\title{
ON REGULAR SET SYSTEMS CONTAINING REGULAR SUBSYSTEMS
}

\author{
AMIN BAHMANIAN AND SADEGHEH HAGHSHENAS
}

\begin{abstract}
Let $X, Y$ be finite sets, $r, s, h, \lambda \in \mathbb{N}$ with $s \geqslant r, X \subsetneq Y$. By $\lambda\left(\begin{array}{l}X \\ h\end{array}\right)$ we mean the collection of all $h$-subsets of $X$ where each subset occurs $\lambda$ times. A coloring of $\lambda\left(\begin{array}{l}X \\ h\end{array}\right)$ is $r$-regular if in every color class each element of $X$ occurs $r$ times. A one-regular color class is a perfect matching. We are interested in the necessary and sufficient conditions under which an $r$-regular coloring of $\lambda\left(\begin{array}{l}X \\ h\end{array}\right)$ can be embedded into an s-regular coloring of $\lambda\left(\begin{array}{l}Y \\ h\end{array}\right)$. Using algebraic techniques involving glueing together orbits of a suitably chosen cyclic group, the first author and Newman (Combinatorica 38 (2018), no. 6, 1309-1335) solved the case when $\lambda=1, r=s, \operatorname{gcd}(|X|,|Y|, h)=\operatorname{gcd}(|Y|, h)$. Using purely combinatorial techniques, we nearly settle the case $h=4$. Two major challenges include finding all the necessary conditions, and obtaining the exact bound for $|Y|$.

It is worth noting that completing partial symmetric latin squares is closely related to the case $\lambda=r=s=1, h=2$ which was solved by Cruse (J. Comb. Theory Ser. A 16 (1974), 18-22).
\end{abstract}

\section{INTRODUCTION}

Consider the following $7 \times 18$ array containing all the 1264 -subsets of $\{1,2, \ldots, 9\}$.

$12351246 \quad 3456|126813671457234823784578 \quad 5678| 12891489167923492569345937895679$ $1234135624561348135716782467247825683578 \quad 14691479156923592689278934793589$ $1245134623561368146814782357256725783478 \quad 12391259134924893579467956896789$ 125613452346127813471568235824573678467814591589178923692479257934693689 123614562345124713781578236826783457456813591379157923892469267945894689 123712481257146715672367245834583468356812691369138923792459456947895789 $1238125812671358145823472468346735674567 \mid 12491279168925893489356936794579$

In each row of this array, every element of $\{1, \ldots, 9\}$ appears exactly eight times. The $7 \times 10$ left subarray contains all the 704 -subsets of $\{1, \ldots, 8\}$, and in each row of this subarray every element of $\{1, \ldots, 8\}$ appears exactly five times. But there is more! The $5 \times 3$ top left subarray contains all the 154 -subsets of $\{1, \ldots, 6\}$, and in each row of this small subarray every element of $\{1, \ldots, 6\}$ appears exactly twice. Figure 1 provides a visual representation of this example, where colored 4-cycles correspond to 4-subsets. The goal of this paper is to construct such highly regular set systems. First, we need to provide some background.

Let $\mathcal{G}:=\lambda K_{m}^{h}$ be the collection of all $h$-subsets of a set $[m]:=\{1, \ldots, m\}$ of vertices where each subset occurs $\lambda$ times. A q-coloring of $\mathcal{G}$ is a mapping $f: \mathcal{G} \rightarrow[q]$; elements of a color $j \in[q]$, written $\mathcal{G}(j)$, form the color class $j$ and the order of $\mathcal{G}(j)$ is $\left|\bigcup_{e \in \mathcal{G}(j)} e\right|$. A coloring is $r$-regular if in each color class the degree (i.e. the number of occurrences) of each vertex

Date: September 23, 2020.

2010 Mathematics Subject Classification. 05C70, 05C65, 05C15.

Key words and phrases. embedding, factorization, edge-coloring, decomposition, Baranyai's theorem, amalgamation, detachment. 
is $r$. The problem of finding regular colorings of $\lambda K_{m}^{h}$ dates back to the 18 th century. Two celebrated examples are the Sylvester's problem that asks for a one-regular coloring of $K_{m}^{h}$ in which each color class is of order $m$, and Steiner's problem that asks for an $\left(\begin{array}{l}r-1 \\ h-1\end{array}\right)$-regular coloring of $K_{m}^{h}$ in which each color class is of order $r$. Sylvester's problem was settled in the 70s by Baranyai [4], and Steiner's problem was solved very recently for large $m$ by Keevash [10] and Glock et al. [8].

An $r$-factorization of $\lambda K_{m}^{h}$ is an $r$-regular coloring in which each color class is of order $m$. In this paper, we are concerned with the following embedding analogue of regular colorings of $\lambda K_{m}^{h}$ which is closely related to Cameron's problem (see [6, Question 1.2].

Problem 1. Find all values of $s$ and $n$ such that the given $r$-factorization of $\mathcal{G}:=\lambda K_{m}^{h}$ can be extended to an s-factorization of $\lambda K_{n}^{h}$.

Previously, Problem 1 was solved for the following cases: $\lambda=r=s=1, h=2$ [7] (this case is closely related to completing partial symmetric latin squares), $\lambda=1, h=2$ [12], $\lambda=r=s=1$ [9], $\lambda=1, h=3$ [2], and $\lambda=1, r=s, \operatorname{gcd}(m, n, h)=\operatorname{gcd}(n, h)$ [3]. The major obstacle for the case where $h \geqslant 3$ stems from the natural difficulty of generalizing graph theoretic results to hypergraphs. In this paper we nearly settle Problem 1 for the case when $h=4$.

Theorem 1.1. If $r\left(\begin{array}{c}n-1 \\ 3\end{array}\right)>s\left(\begin{array}{c}m-1 \\ 3\end{array}\right)$, then an r-factorization of $\lambda K_{m}^{4}$ can be extended to an $s$-factorization of $\lambda K_{n}^{4}$ if and only if

$$
\begin{gathered}
4|r m, \quad 4| s n, \quad r\left|\lambda\left(\begin{array}{c}
m-1 \\
3
\end{array}\right), \quad s\right| \lambda\left(\begin{array}{c}
n-1 \\
3
\end{array}\right), \quad 1 \leqslant \frac{s}{r} \leqslant\left(\begin{array}{c}
n-1 \\
3
\end{array}\right) /\left(\begin{array}{c}
m-1 \\
3
\end{array}\right) ; \\
n \geqslant\left\{\begin{array}{c}
2 m \quad \text { if } s=r \\
\frac{4 m}{3} \quad \text { if } s>r
\end{array} ;\right. \\
(n-m)\left(\begin{array}{c}
m \\
3
\end{array}\right) \geqslant\left(m-\frac{n}{2}\right)\left[\left(\begin{array}{c}
n-1 \\
3
\end{array}\right)-\frac{s}{r}\left(\begin{array}{c}
m-1 \\
3
\end{array}\right)\right] ; \\
2(n-m)\left(\begin{array}{c}
m \\
3
\end{array}\right)+\left(\begin{array}{c}
m \\
2
\end{array}\right)\left(\begin{array}{c}
n-m \\
2
\end{array}\right) \geqslant\left(m-\frac{n}{4}\right)\left[\left(\begin{array}{c}
n-1 \\
3
\end{array}\right)-\frac{s}{r}\left(\begin{array}{c}
m-1 \\
3
\end{array}\right)\right] .
\end{gathered}
$$

Theorem 1.2. If $n \geqslant 4 m / 3$ and $r\left(\begin{array}{c}n-1 \\ 3\end{array}\right)=s\left(\begin{array}{c}m-1 \\ 3\end{array}\right)$, then an r-factorization of $\lambda K_{m}^{4}$ can be extended to an s-factorization of $\lambda K_{n}^{4}$ if and only if (1) holds.

We note that none of the previous partial solutions to Problem 1 considered $\lambda>1$. Two major challenges in proving Theorems 1.1 and 1.2 include finding all the necessary conditions (see Section 2), and obtaining the exact bound for $n$. Judging from the literature on embedding results for other combinatorial structures such as latin squares and Steiner triple systems, finding a sharp lower bound for $n$ in general is quite difficult. A noteworthy example is Lindner's conjecture [11] that any partial Steiner triple system of order $m$ can be embedded in a Steiner triple system of order $n$ if $n \equiv 1,3(\bmod 6)$ and $n \geqslant 2 m+1$, which despite numerous attempts, took over thirty years to be resolved [5].

The layout of the paper is as follows. In Section 2, we discuss the necessary conditions. In Section 3 we provide the sketch of the proof of sufficiency. Coloring the edges is divided into two parts, and the easier part is provided in Section 4. Several crucial inequalities will 
be needed before we are able to complete the coloring in Section 5, and those are proven in Section 6.

We end this section with some notation. A hypergraph $\mathcal{G}$ is a pair $(V(\mathcal{G}), E(\mathcal{G})$ ) where $V(\mathcal{G})$ is a finite set called the vertex set, $E(\mathcal{G})$ is the edge multiset, where every edge is itself a multi-subset of $V(\mathcal{G})$. This means that not only can an edge occur multiple times in $E(\mathcal{G})$, but also each vertex can have multiple occurrences within an edge. The total number of occurrences of a vertex $v$ among all edges of $E(\mathcal{G})$ is called the degree, $\operatorname{deg}_{\mathcal{G}}(v)$ of $v$ in $\mathcal{G}$. For two hypergraphs $\mathcal{G}$ and $\mathcal{F}, \mathcal{G} \backslash \mathcal{F}$ is the hypergraph whose vertex set is $V(\mathcal{G})$ and whose edge set is $E(\mathcal{G}) \backslash E(\mathcal{F})$.

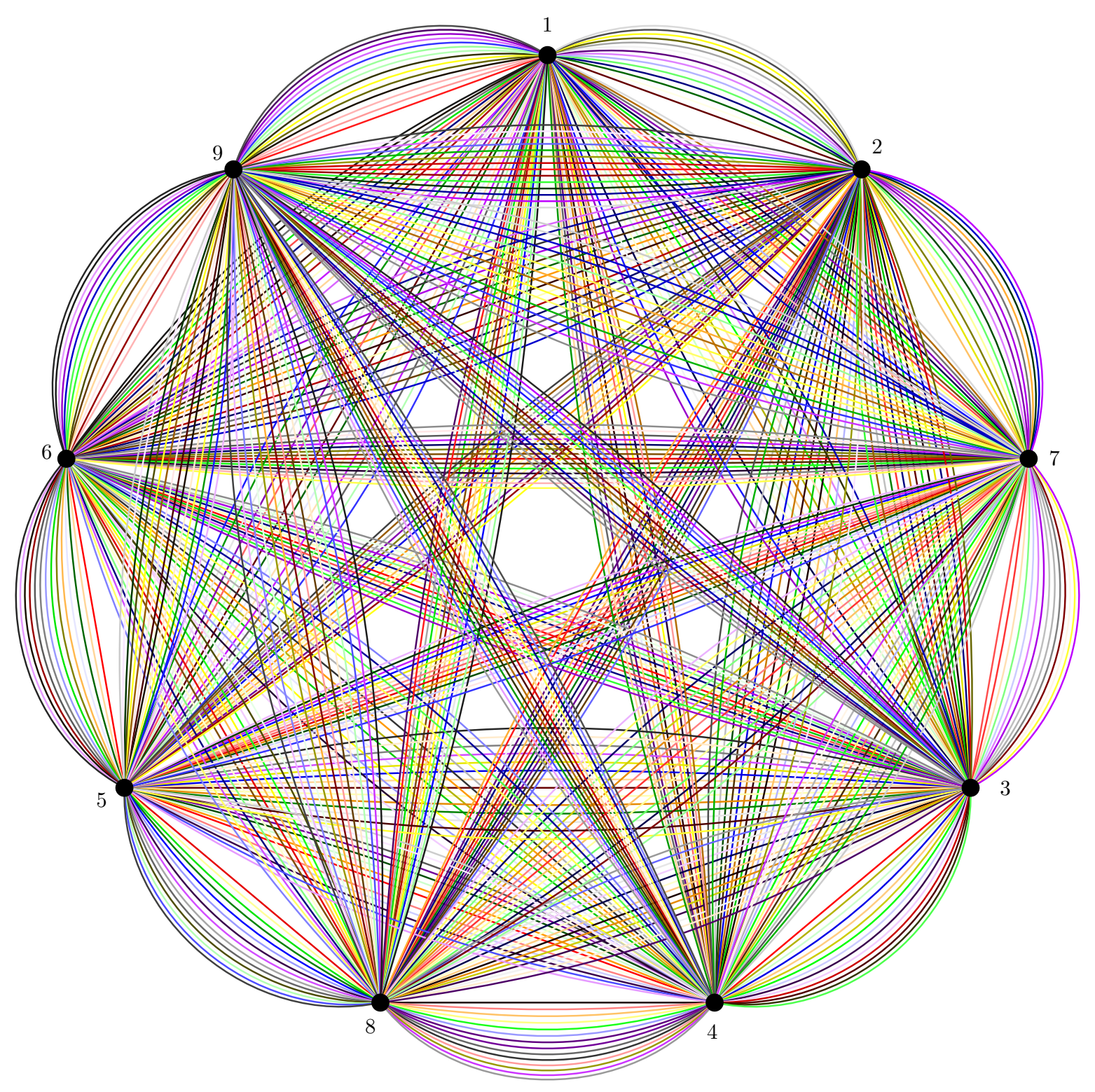

FiguRE 1. A 2-factorization of $K_{6}^{4}$ embedded into a 5-factorization of $K_{8}^{4}$ which is embedded into an 8-factorization of $K_{9}^{4}$ 


\section{Necessary Conditions}

In order to avoid trivial cases, we shall make the following assumptions: (i) $n>m \geqslant 4$, (ii) if $m=4$, then $\lambda \geqslant 2$ and $r \geqslant 2$.

The following lemma will be used without further explanation when required.

Lemma 2.1. For $m, n \in \mathbb{N}$ with $n>m$, we have

(a) $\left(\begin{array}{l}n \\ 4\end{array}\right)=\left(\begin{array}{c}m \\ 4\end{array}\right)+(n-m)\left(\begin{array}{c}m \\ 3\end{array}\right)+\left(\begin{array}{c}m \\ 2\end{array}\right)\left(\begin{array}{c}n-m \\ 2\end{array}\right)+m\left(\begin{array}{c}n-m \\ 3\end{array}\right)+\left(\begin{array}{c}n-m \\ 4\end{array}\right)$,

(b) $\left(\begin{array}{c}n-1 \\ 3\end{array}\right)=\left(\begin{array}{c}m-1 \\ 3\end{array}\right)+(n-m)\left(\begin{array}{c}m-1 \\ 2\end{array}\right)+(m-1)\left(\begin{array}{c}n-m \\ 2\end{array}\right)+\left(\begin{array}{c}n-m \\ 3\end{array}\right)$, and

(c) $m\left[\left(\begin{array}{c}n-1 \\ 3\end{array}\right)-\left(\begin{array}{c}m-1 \\ 3\end{array}\right)\right]=3(n-m)\left(\begin{array}{c}m \\ 3\end{array}\right)+2\left(\begin{array}{c}m \\ 2\end{array}\right)\left(\begin{array}{c}n-m \\ 2\end{array}\right)+m\left(\begin{array}{c}n-m \\ 3\end{array}\right)$.

The proof is based on a simple double counting argument, and we shall skip it here.

A triple $(m, r, \lambda)$ is admissible if $4 \mid r m$ and $r \mid \lambda\left(\begin{array}{c}m-1 \\ 3\end{array}\right)$. The following lemma settles the necessary conditions.

Lemma 2.2. If an r-factorization of $\lambda K_{m}^{4}$ can be extended to an s-factorization of $\lambda K_{n}^{4}$, then the following conditions hold.

(N1) The triples $(m, r, \lambda)$ and $(n, s, \lambda)$ are admissible;

(N2) $1 \leqslant s / r \leqslant\left(\begin{array}{c}n-1 \\ 3\end{array}\right) /\left(\begin{array}{c}m-1 \\ 3\end{array}\right)$;

(N3) If $s=r$, then $n \geqslant 2 m$;

(N4) $n \geqslant \frac{m}{3}(4-r / s)$;

(N5) If $1<s / r<\left(\begin{array}{c}n-1 \\ 3\end{array}\right) /\left(\begin{array}{c}m-1 \\ 3\end{array}\right)$, then $n \geqslant 4 m / 3$;

(N6)

$$
(n-m)\left(\begin{array}{c}
m \\
3
\end{array}\right) \geqslant\left(m-\frac{n}{2}\right)\left[\left(\begin{array}{c}
n-1 \\
3
\end{array}\right)-\frac{s}{r}\left(\begin{array}{c}
m-1 \\
3
\end{array}\right)\right]
$$

$$
2(n-m)\left(\begin{array}{c}
m \\
3
\end{array}\right)+\left(\begin{array}{c}
m \\
2
\end{array}\right)\left(\begin{array}{c}
n-m \\
2
\end{array}\right) \geqslant\left(m-\frac{n}{4}\right)\left[\left(\begin{array}{c}
n-1 \\
3
\end{array}\right)-\frac{s}{r}\left(\begin{array}{c}
m-1 \\
3
\end{array}\right)\right]
$$

(N8) If $s / r=\left(\begin{array}{c}n-1 \\ 3\end{array}\right) /\left(\begin{array}{c}m-1 \\ 3\end{array}\right)$, then

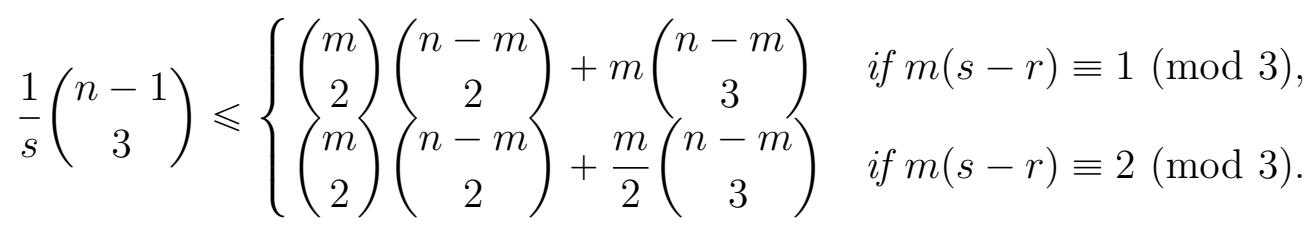

Proof. Suppose that an $r$-factorization of $\lambda K_{m}^{4}$ is extended to an $s$-factorization of $\lambda K_{n}^{4}$. Since $\lambda K_{m}^{4}$ is $r$-factorable, $r$ divides the degree of each vertex in $\lambda K_{m}^{4}$. The existence of an $r$-factor in $\lambda K_{m}^{4}$ implies that $4 \mid r m$. Thus, $(m, r, \lambda)$ is admissible. A similar argument shows that $(n, s, \lambda)$ is also admissible. Consequently, $q:=\lambda\left(\begin{array}{c}m-1 \\ 3\end{array}\right) / r$ and $k:=\lambda\left(\begin{array}{c}n-1 \\ 3\end{array}\right) / s$ are integers.

In order to extend an $r$-factorization of $\lambda K_{m}^{4}$ to an $s$-factorization of $\lambda K_{n}^{4}$, we must clearly have $s \geqslant r$. Moreover, the number of colors used in an $r$-factorization of $\lambda K_{m}^{4}, q$, is no more than the number of colors used in an $s$-factorization of $\lambda K_{n}^{4}, k$, and so (N2) holds.

For the rest of the proof, we shall refer to the $m$ vertices of $\lambda K_{m}^{4} \subseteq \lambda K_{n}^{4}$, and the remaining $n-m$ vertices of $\lambda K_{n}^{4} \backslash \lambda K_{m}^{4}$ as the old vertices, and the new vertices, respectively. Moreover, $\kappa:=\kappa_{1} \cup \kappa_{2}$ where $\kappa_{1}:=\{1, \ldots, q\}$ is the set of the old colors (those used in the coloring of $\lambda K_{m}^{4}$ ), and $\kappa_{2}:=\{q+1, \ldots, k\}$ is the set of the new colors (those used only in the coloring of $\left.\lambda K_{n}^{4} \backslash \lambda K_{m}^{4}\right)$. For $j \in \kappa$, let $a_{j}, e_{j}, f_{j}, g_{j}$, and $\ell_{j}$ be the number of edges colored $j$ in $\lambda K_{n}^{4}$ that are incident with exactly $0,1,2,3$, and 4 new vertices, respectively. 
To prove (N3) suppose that $s=r$, and let $j \in \kappa_{1}$. We cannot have any edges colored $j$ between the old vertices and the new vertices. Therefore, to form an $r$-factor, we can only use the new edges, and so $n-m \geqslant 4$. In order to form an $r$-factor in $\lambda K_{n}^{4}$ with color $j$, we need to have $r(n-m) / 4$ edges colored $j$ between the new vertices. Hence,

$$
\lambda\left(\begin{array}{c}
n-m \\
4
\end{array}\right) \geqslant q \frac{r(n-m)}{4} .
$$

This implies $\frac{4}{n-m}\left(\begin{array}{c}n-m \\ 4\end{array}\right) \geqslant\left(\begin{array}{c}m-1 \\ 3\end{array}\right)$. Therefore, $\left(\begin{array}{c}n-m-1 \\ 3\end{array}\right) \geqslant\left(\begin{array}{c}m-1 \\ 3\end{array}\right)$, or equivalently, $n-m-1 \geqslant$ $m-1$, and so (N3) is satisfied.

In an $s$-factorization of $\lambda K_{n}^{h}$, each of the new vertices is adjacent with exactly $s$ edges of each color, so all the new vertices are adjacent with at most $s(n-m)$ edges of each color. We have $s(n-m)+a_{j} \geqslant s n / 4$ for $j \in \kappa$. Since $a_{j}=r m / 4$ for $j \in \kappa_{1}$, we have $s(n-m)+r m / 4 \geqslant s n / 4$, which proves (N4). Moreover, if $k>q$, then $\kappa_{2} \neq \varnothing$ and since $a_{j}=0$ for $j \in \kappa_{2}$, we have $s(n-m) \geqslant s n / 4$ which proves (N5).

To prove (N6) and (N7), observe that for $k=q$, the right hand side of (N6) and (N7) is zero, and there is nothing to prove. So let us assume that $k>q$. Within each new color class of $\lambda K_{n}^{4}$, the degree sum of all the old vertices is $s m$. Therefore, for any $j \in \kappa_{2}$,

$$
s m=3 e_{j}+2 f_{j}+g_{j} .
$$

Since the number of edges in each new color class of $\lambda K_{n}^{4}$ is $s n / 4$, we have

$$
s n / 4=e_{j}+f_{j}+g_{j}+\ell_{j} .
$$

Therefore,

$$
s m-\frac{s n}{4}=2 e_{j}+f_{j}-\ell_{j} \leqslant 2 e_{j}+f_{j} .
$$

By taking the sum over all new colors, we have

$$
(k-q)\left(s m-\frac{s n}{4}\right) \leqslant 2 \lambda(n-m)\left(\begin{array}{c}
m \\
3
\end{array}\right)+\lambda\left(\begin{array}{c}
m \\
2
\end{array}\right)\left(\begin{array}{c}
n-m \\
2
\end{array}\right),
$$

which proves (N7). Similarly, we have

$$
s m-\frac{s n}{2}=e_{j}-g_{j}-2 \ell_{j} \leqslant e_{j},
$$

and so

$$
(k-q)\left(s m-\frac{s n}{2}\right) \leqslant \lambda(n-m)\left(\begin{array}{c}
m \\
3
\end{array}\right)
$$

which proves (N6).

To prove (N8), first we show that

$$
\text { If } k=q \text {, and } m(s-r) \not \equiv 0(\bmod 3) \text {, then } n \geqslant m+2 \text {. }
$$

Suppose by the contrary that $n=m+1$. Since $k=q$, we have $\left(\begin{array}{c}m \\ 3\end{array}\right) / s=\left(\begin{array}{c}m-1 \\ 3\end{array}\right) / r$, and so $m(s-r)=3 s$, which is a contradiction. Within the new edges of $\lambda K_{n}^{4}$, the degree sum of all the old vertices in each old color class is $m(s-r)$. Therefore,

$$
m(s-r)=3 e_{j}+2 f_{j}+g_{j} \equiv 2 f_{j}+g_{j}(\bmod 3) \text { for } j \in \kappa_{1} .
$$

There are two cases to consider. 
(a) If $m(s-r) \equiv 1(\bmod 3)$, then $2 f_{j}+g_{j} \equiv 1(\bmod 3)$, and so $f_{j}+g_{j} \geqslant 1$ for each $j \in \kappa_{1}$. Hence,

$$
\lambda\left(\begin{array}{c}
m \\
2
\end{array}\right)\left(\begin{array}{c}
n-m \\
2
\end{array}\right)+\lambda m\left(\begin{array}{c}
n-m \\
3
\end{array}\right) \geqslant q .
$$

(b) If $m(s-r) \equiv 2(\bmod 3)$, then $2 f_{j}+g_{j} \equiv 2(\bmod 3)$, and so $2 f_{j}+g_{j} \geqslant 2$ for each $j \in \kappa_{1}$. Hence,

$$
2 \lambda\left(\begin{array}{c}
m \\
2
\end{array}\right)\left(\begin{array}{c}
n-m \\
2
\end{array}\right)+\lambda m\left(\begin{array}{c}
n-m \\
3
\end{array}\right) \geqslant 2 q
$$

Although, we will need the necessary conditions (N4) and (N8), in the next two lemmas, we show that (N4) and (N8) are redundant.

Lemma 2.3. (N5) implies (N4).

Proof. If $k>q$, then by (N5), $n \geqslant 4 m / 3$ and so (N4) clearly holds. Let us assume that $k=q$. We need to show that $n \geqslant \frac{m}{3}\left(4-\left[\left(\begin{array}{c}m-1 \\ 3\end{array}\right) /\left(\begin{array}{c}n-1 \\ 3\end{array}\right)\right]\right)$, or equivalently,

$$
3 n\left(\begin{array}{c}
n-1 \\
3
\end{array}\right)-4 m\left(\begin{array}{c}
n-1 \\
3
\end{array}\right)+m\left(\begin{array}{c}
m-1 \\
3
\end{array}\right) \geqslant 0 .
$$

Since $n \geqslant m+1$ and $m \geqslant 4$, we have

$$
\begin{aligned}
& 9 n\left(\begin{array}{c}
n-1 \\
3
\end{array}\right)-12 m\left(\begin{array}{c}
n-1 \\
3
\end{array}\right)+3 m\left(\begin{array}{c}
m-1 \\
3
\end{array}\right) \\
= & 36\left(\begin{array}{l}
n \\
4
\end{array}\right)-12 m\left(\begin{array}{c}
n-1 \\
3
\end{array}\right)+12\left(\begin{array}{c}
m \\
4
\end{array}\right) \\
= & 12\left[\left(\begin{array}{c}
m \\
2
\end{array}\right)\left(\begin{array}{c}
n-m \\
2
\end{array}\right)+2 m\left(\begin{array}{c}
n-m \\
3
\end{array}\right)+3\left(\begin{array}{c}
n-m \\
4
\end{array}\right)\right] \\
= & \left(\begin{array}{c}
n-m \\
2
\end{array}\right)\left(n(3 n+2 m-15)+m^{2}-7 m+18\right) \\
\geqslant & \left(\begin{array}{c}
n-m \\
2
\end{array}\right)(3(m+1)+2 m-15) \geqslant 0 .
\end{aligned}
$$

Lemma 2.4. If $m, n, r, s \in \mathbb{N}$ such that $n>m, s>r$, and $s / r=\left(\begin{array}{c}n-1 \\ 3\end{array}\right) /\left(\begin{array}{c}m-1 \\ 3\end{array}\right)$, then

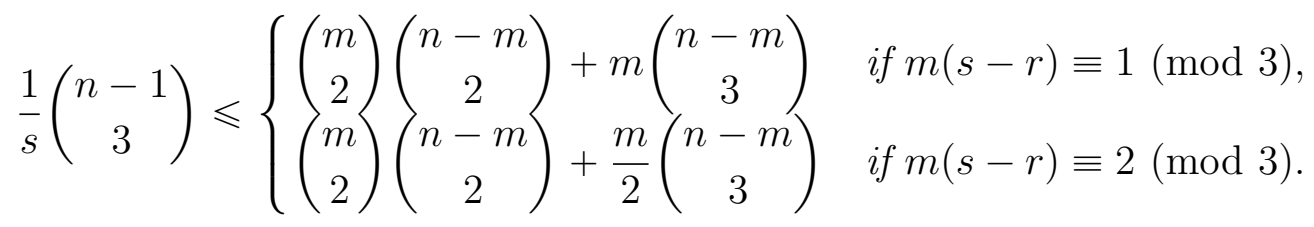

Proof. By (5), $n \geqslant m+2$. We show that

$$
\text { If } k=q \text {, and } n=m+2 \text {, then } s \geqslant r+2 \text {. }
$$

Suppose on the contrary that $k=q, n=m+2$, but $s=r+1$. Since $s / r=\left(\begin{array}{c}n-1 \\ 3\end{array}\right) /\left(\begin{array}{c}m-1 \\ 3\end{array}\right)$, we have $s(m-2)(m-3)=r m(m+1)$, or equivalently, $m^{2}-(6 r+5) m+6(r+1)=0$. This implies that $m=\left(6 r+5 \pm \sqrt{36 r^{2}+36 r+1}\right) / 2$. Since $(6 r+2)^{2}<36 r^{2}+36 r+1<(6 r+3)^{2}$, $36 r^{2}+36 r+1$ is not a perfect square, but $m$ is an integer, this is a contradiction. 
Since $s / r=\left(\begin{array}{c}n-1 \\ 3\end{array}\right) /\left(\begin{array}{c}m-1 \\ 3\end{array}\right)$, we have

$$
\begin{aligned}
\frac{s}{r}\left(\begin{array}{c}
m-1 \\
3
\end{array}\right) & =\left(\begin{array}{c}
n-1 \\
3
\end{array}\right) \\
& =\left(\begin{array}{c}
m-1 \\
3
\end{array}\right)+(n-m)\left(\begin{array}{c}
m-1 \\
2
\end{array}\right)+(m-1)\left(\begin{array}{c}
n-m \\
2
\end{array}\right)+\left(\begin{array}{c}
n-m \\
3
\end{array}\right) .
\end{aligned}
$$

Therefore,

$$
\left(\begin{array}{c}
m-1 \\
3
\end{array}\right)=\frac{r}{s-r}\left[(n-m)\left(\begin{array}{c}
m-1 \\
2
\end{array}\right)+(m-1)\left(\begin{array}{c}
n-m \\
2
\end{array}\right)+\left(\begin{array}{c}
n-m \\
3
\end{array}\right)\right]
$$

To complete the proof, there are two cases to consider.

(i) If $m(s-r) \equiv 1(\bmod 3)$, we need to show that

$$
\begin{aligned}
r m\left(\begin{array}{c}
n-m \\
3
\end{array}\right)+ & r\left(\begin{array}{c}
m \\
2
\end{array}\right)\left(\begin{array}{c}
n-m \\
2
\end{array}\right) \\
& \geqslant \frac{r}{s-r}\left[(n-m)\left(\begin{array}{c}
m-1 \\
2
\end{array}\right)+(m-1)\left(\begin{array}{c}
n-m \\
2
\end{array}\right)+\left(\begin{array}{c}
n-m \\
3
\end{array}\right)\right],
\end{aligned}
$$

or, equivalently

$[m(s-r)-1]\left(\begin{array}{c}n-m \\ 3\end{array}\right)+[m(s-r)-2] \frac{m-1}{2}\left(\begin{array}{c}n-m \\ 2\end{array}\right) \geqslant(n-m)\left(\begin{array}{c}m-1 \\ 2\end{array}\right)$.

If $n-m=2$, then $s-r \geqslant 2$, and so it is enough to show that $(2 m-2) \frac{m-1}{2} \geqslant 2\left(\begin{array}{c}m-1 \\ 2\end{array}\right)$, which is clearly true. If $n-m \geqslant 3$, since $s-r \geqslant 1$, it is enough to show that $(m-1)\left(\begin{array}{c}n-m \\ 3\end{array}\right)+\left(\begin{array}{c}m-1 \\ 2\end{array}\right)\left(\begin{array}{c}n-m \\ 2\end{array}\right) \geqslant(n-m)\left(\begin{array}{c}m-1 \\ 2\end{array}\right)$, or equivalently,

$$
\left(\begin{array}{c}
n-m \\
2
\end{array}\right)\left[(m-1)(n-m-2)+3\left(\begin{array}{c}
m-1 \\
2
\end{array}\right)\right] \geqslant 3(n-m)\left(\begin{array}{c}
m-1 \\
2
\end{array}\right) .
$$

Since $n-m \geqslant 3$, we have $\left(\begin{array}{c}n-m \\ 2\end{array}\right) \geqslant n-m$. Therefore, it is enough to show that

$$
(m-1)(n-m-2)+3\left(\begin{array}{c}
m-1 \\
2
\end{array}\right) \geqslant 3\left(\begin{array}{c}
m-1 \\
2
\end{array}\right)
$$

which clearly holds.

(ii) If $m(s-r) \equiv 2(\bmod 3)$, we need to show that

$$
[m(s-r)-2]\left(\begin{array}{c}
n-m \\
2
\end{array}\right)\left(\frac{n+2 m-5}{3}\right) \geqslant 2(n-m)\left(\begin{array}{c}
m-1 \\
2
\end{array}\right) .
$$

If $n-m=2$, then $s-r \geqslant 2$, and so it is enough to show that $(2 m-2) \frac{3 m-3}{3} \geqslant 4\left(\begin{array}{c}m-1 \\ 2\end{array}\right)$, which is clearly true. If $n-m \geqslant 3$, then we have $\left(\begin{array}{c}n-m \\ 2\end{array}\right) \geqslant n-m$. Therefore, since $s-r \geqslant 1$, it suffices to show that $(n+2 m-5) / 3 \geqslant m-1$. This is equivalent to $n-m \geqslant 2$, which is trivial.

Remark 2.5. Neither Condition (3) nor Condition (4) can be eliminated from Theorem 1.1. For example, a 4-factorization of $K_{7}^{4}$ cannot be extended to a 6 -factorization of $K_{10}^{4}$, and a 6 -factorization of $K_{10}^{4}$ cannot be extended to a 7 -factorization of $K_{16}^{4}$. In the first example all conditions but (3) hold, and in the second example all conditions but (4) hold. 


\section{Sketch of Proof}

Throughout the rest of this paper, we shall assume that the conditions (1)-(4) (or equivalently, (N1)-(N8)) hold, $n \geqslant 4 m / 3$, and that

$$
q:=\frac{\lambda}{r}\left(\begin{array}{c}
m-1 \\
3
\end{array}\right), \quad k:=\frac{\lambda}{s}\left(\begin{array}{c}
n-1 \\
3
\end{array}\right) .
$$

Since $(m, r, \lambda)$ and $(n, s, \lambda)$ are admissible, both $q$ and $k$ are integers. Let

$$
\kappa_{1}=\{1, \ldots, q\}, \quad \kappa_{2}=\{q+1, \ldots, k\}, \quad \kappa=\kappa_{1} \cup \kappa_{2} .
$$

Let $\mathcal{F}$ be a 2-vertex hypergraph with $V(\mathcal{F})=\{u, v\}$. In order to describe the edge set of $\mathcal{F}$, first we need to introduce some notation. The multiplicity of an edge $e$ in $\mathcal{F}$, written mult $(e)$, is the number of repetitions of $e$ in $\mathcal{F}$. A $u^{i} v^{j}$-edge is an edge in which vertex $u$ occurs $i$ times and vertex $v$ occurs $j$ times. When we color the edges of $\mathcal{F}$, we use $\operatorname{deg}_{j}(v)$ and $\operatorname{mult}_{j}(e)$ for the degree of $v$, and the multiplicity of $e$ in color class $j$. The following describes the edge set of $\mathcal{F}$.

$$
\operatorname{mult}\left(u^{i} v^{4-i}\right)=\lambda\left(\begin{array}{c}
m \\
i
\end{array}\right)\left(\begin{array}{c}
n-m \\
4-i
\end{array}\right) \quad \text { for } 0 \leqslant i \leqslant 3
$$

Observe that $\mathcal{F}$ can be obtained by identifying all the $m$ old vertices of $\mathcal{G}:=\lambda K_{n}^{4} \backslash \lambda K_{m}^{4}$ by a vertex $u$, and identifying all the remaining $n-m$ new vertices with $v$. We say that $\mathcal{F}$ is an amalgamation of $\mathcal{G}$, and that $\mathcal{G}$ is a detachment of $\mathcal{F}$.

We think of the given $r$-factorization of $\lambda K_{m}^{4}$ as a $q$-coloring of $\lambda K_{m}^{4}$ in which each color class induces an $r$-factor. In order to extend the $r$-factorization of $\lambda K_{m}^{4}$ to an $s$-factorization of $\lambda K_{n}^{4}$, we need to color $\mathcal{G}$ with $k$ colors such that each color class of $\lambda K_{n}^{4}$ induces an $s$-factor. If we can obtain such a coloring, then in the amalgamation $\mathcal{F}$ of $\mathcal{G}, \operatorname{deg}_{j}(u)=m(s-r)$ for $j \in \kappa_{1}, \operatorname{deg}_{j}(u)=s m$ for $j \in \kappa_{2}$, and $\operatorname{deg}_{j}(v)=s(n-m)$ for $j \in \kappa$. More importantly, by the following lemma which is an immediate consequence of a result of the first author (see [1, Theorem 4.1]), the converse of the previous statement is also true.

Lemma 3.1. If the hypergraph $\mathcal{F}$ described in $(7)$ can be colored so that

$$
\operatorname{deg}_{j}(x)= \begin{cases}m(s-r) & \text { if } x=u, j \in \kappa_{1}, \\ s m & \text { if } x=u, j \in \kappa_{2}, \\ s(n-m) & \text { if } x=v, j \in \kappa,\end{cases}
$$

then an r-factorization of $\lambda K_{m}^{4}$ can be extended to an s-factorization of $\lambda K_{n}^{4}$.

Thus, the problem of extending an $r$-factorization of $\lambda K_{m}^{4}$ to an $s$-factorization of $\lambda K_{n}^{4}$ is reduced to coloring of the amalgamation $\mathcal{F}$ of $\mathcal{G}$. Although it is much easier to color $\mathcal{F}$ than to color $\mathcal{G}$, it is particularly very difficult to color $\mathcal{F}$ when $n$ and $m$ are very close to each other. The rest of the paper is devoted to a coloring of $\mathcal{F}$ ensuring that (8) is satisfied. As we shall see in the next section, provided we carefully color the $u^{3} v$-edges and $u^{2} v^{2}$-edges, coloring the remaining edges of $\mathcal{F}$ is straightforward (see Lemma 4.1). Before we can color the $u^{3} v$-edges and $u^{2} v^{2}$-edges, we need to prove several crucial inequalities. We shall do this in Section 6 following the coloring of the $u^{3} v$-edges and $u^{2} v^{2}$-edges in Section 5 . 


\section{Colorings I}

In this section we will show that if we can color the $u^{3} v$-edges and $u^{2} v^{2}$-edges so that certain conditions are met (see Condition (9)), then it is easy to color the $u v^{3}$-edges and $v^{4}$-edges. To achieve this goal, let us introduce the following fixed parameters.

$$
\left\{\begin{array}{rlrl}
\iota_{1}:=s m-\frac{s n}{2}-\frac{r m}{2}, & & \\
\iota_{2}:=s m-\frac{s n}{2}, & & \text { if } \kappa_{2} \neq \varnothing, \\
\rho_{1}:=\frac{s m}{3}-\frac{r m}{3}, & & \text { if } \kappa_{2} \neq \varnothing, \\
\rho_{2}:=\frac{s m}{3}, & \\
\rho_{1}^{\prime}:=\frac{s m}{2}-\frac{s n}{8}-\frac{3 r m}{8}, & \\
\rho_{2}^{\prime}:=\frac{s m}{2}-\frac{s n}{8}, & \text { if } \kappa_{2} \neq \varnothing .
\end{array}\right.
$$

Since by $(\mathrm{N} 1),(m, r, \lambda)$ and $(n, s, \lambda)$ are admissible, $\iota_{1}$ and $\iota_{2}$ are integers. By (N2), $s \geqslant r \geqslant 1$, and so we have that $\rho_{1} \geqslant 0$ and $\rho_{2}>0$. Observe that $\iota_{1}$ and $\iota_{2}$ are not necessarily non-negative. In fact, $\iota_{1} \geqslant 0$ if an only if $n \leqslant\left(2-\frac{r}{s}\right) m$, and $\iota_{2} \geqslant 0$ if and only if $n \leqslant 2 m$. In addition, $\rho_{1}, \rho_{2}, \rho_{1}^{\prime}, \rho_{2}^{\prime}$ are not necessarily integers. It is also easy to see that $\rho_{1}^{\prime} \geqslant 0$ if and only if $n \leqslant\left(4-\frac{3 r}{s}\right) m$, and $\rho_{2}^{\prime} \geqslant 0$ if and only if $n \leqslant 4 m$. By (N4), $\iota_{1} \leqslant \rho_{1}^{\prime} \leqslant \rho_{1}$. Finally, by (N5) for $k>q, n \geqslant 4 m / 3$, so we have that $\iota_{2} \leqslant \rho_{2}^{\prime} \leqslant \rho_{2}$ (for $k>q$ ).

Once we color the $u^{3} v$-edges, we can introduce the following further parameters.

$$
\left\{\begin{aligned}
\iota_{1 j}:=s m-\frac{s n}{4}-2 \operatorname{mult}_{j}\left(u^{3} v\right)-\frac{3 r m}{4}, & & j \in \kappa_{1} \\
\iota_{2 j}:=s m-\frac{s n}{4}-2 \operatorname{mult}_{j}\left(u^{3} v\right), & & j \in \kappa_{2} \\
\rho_{1 j}:=\frac{s m}{2}-\frac{3}{2} \operatorname{mult}_{j}\left(u^{3} v\right)-\frac{r m}{2}, & & j \in \kappa_{1} \\
\rho_{2 j}:=\frac{s m}{2}-\frac{3}{2} \operatorname{mult}_{j}\left(u^{3} v\right), & & j \in \kappa_{2} .
\end{aligned}\right.
$$

Since $(m, r, \lambda)$ and $(n, s, \lambda)$ are admissible, $\iota_{1 j} \in \mathbb{Z}$ for $j \in \kappa_{1}$, and $\iota_{2 j} \in \mathbb{Z}$ for $j \in \kappa_{2}$. Observe that $\rho_{1 j}$ (for $j \in \kappa_{1}$ ), and $\rho_{2 j}$ (for $j \in \kappa_{2}$ ) are not necessarily integers. Moreover,

(i) for $j \in \kappa_{1}, \rho_{1 j} \geqslant 0$ if and only if $\operatorname{mult}_{j}\left(u^{3} v\right) \leqslant \rho_{1}$,

(ii) for $j \in \kappa_{2}, \rho_{2 j} \geqslant 0$ if and only if $\operatorname{mult}_{j}\left(u^{3} v\right) \leqslant \rho_{2}$,

(iii) for $j \in \kappa_{1}, \iota_{1 j} \geqslant 0$ if and only if $\operatorname{mult}_{j}\left(u^{3} v\right) \leqslant \rho_{1}^{\prime}$,

(iv) for $j \in \kappa_{2}, \iota_{2 j} \geqslant 0$ if and only if $\operatorname{mult}_{j}\left(u^{3} v\right) \leqslant \rho_{2}^{\prime}$,

(v) for $j \in \kappa_{1}, \rho_{1 j} \geqslant \iota_{1 j}$ if and only if $\operatorname{mult}_{j}\left(u^{3} v\right) \geqslant \iota_{1}$, and

(vi) for $j \in \kappa_{2}, \rho_{2 j} \geqslant \iota_{2 j}$ if and only if $\operatorname{mult}_{j}\left(u^{3} v\right) \geqslant \iota_{2}$.

We have summarized all the useful information about $\iota_{1}, \iota_{2}, \rho_{1}, \rho_{2}, \rho_{1}^{\prime}, \rho_{2}^{\prime}, \iota_{1 j}, \rho_{1 j}$ for $j \in \kappa_{1}$, and $\iota_{2 j}, \rho_{2 j}$ for $j \in \kappa_{2}$ in Table 1 .

Lemma 4.1. Suppose that the $u^{3} v$-edges and $u^{2} v^{2}$-edges of $\mathcal{F}$ can be colored such that

$$
\left\{\begin{array}{c}
\iota_{i} \leqslant \operatorname{mult}_{j}\left(u^{3} v\right) \leqslant \rho_{i} \\
\iota_{i j} \leqslant \operatorname{mult}_{j}\left(u^{2} v^{2}\right) \leqslant \rho_{i j}
\end{array}\right\} \quad j \in \kappa_{i}, i=1,2 .
$$


Then the remaining edges of $\mathcal{F}$ can be colored so that (8) holds. Accordingly, if (9) is satisfied, then an r-factorization of $\lambda K_{m}^{4}$ can be extended to an s-factorization of $\lambda K_{n}^{4}$.

Proof. Suppose that the $u^{3} v$-edges and $u^{2} v^{2}$-edges of $\mathcal{F}$ can be colored such that (9) holds. First, we claim that we can color the $u v^{3}$-edges such that

$$
\operatorname{mult}_{j}\left(u v^{3}\right)=2 \rho_{i j}-2 \operatorname{mult}_{j}\left(u^{2} v^{2}\right) \quad j \in \kappa_{i}, i=1,2 .
$$

By $(9), \operatorname{mult}_{j}\left(u^{2} v^{2}\right) \leqslant \rho_{1 j}$ for $j \in \kappa_{1}$, and therefore, mult $_{j}\left(u v^{3}\right) \geqslant 0$ for $j \in \kappa_{1}$. Likewise, mult $_{j}\left(u^{2} v^{2}\right) \leqslant \rho_{2 j}$ for $j \in \kappa_{2}$, and so, $\operatorname{mult}_{j}\left(u v^{3}\right) \geqslant 0$ for $j \in \kappa_{2}$. Moreover, $2 \rho_{i j} \in \mathbb{Z}$ for $j \in \kappa_{i}, i=1,2$, and so mult ${ }_{j}\left(u v^{3}\right) \in \mathbb{Z}$ for $j \in \kappa$. Hence, the following confirms that the coloring of the $u v^{3}$-edges satisfying (10) is possible.

$$
\begin{aligned}
\sum_{j \in \kappa} \operatorname{mult}_{j}\left(u v^{3}\right)= & \sum_{j \in \kappa_{1}} \operatorname{mult}_{j}\left(u v^{3}\right)+\sum_{j \in \kappa_{2}} \operatorname{mult}_{j}\left(u v^{3}\right) \\
= & q m(s-r)-3 \sum_{j \in \kappa_{1}} \operatorname{mult}_{j}\left(u^{3} v\right)-2 \sum_{j \in \kappa_{1}} \operatorname{mult}_{j}\left(u^{2} v^{2}\right) \\
& +s m(k-q)-3 \sum_{j \in \kappa_{2}} \operatorname{mult}_{j}\left(u^{3} v\right)-2 \sum_{j \in \kappa_{2}} \operatorname{mult}_{j}\left(u^{2} v^{2}\right) \\
= & k s m-q r m-3 \lambda(n-m)\left(\begin{array}{c}
m \\
3
\end{array}\right)-2 \lambda\left(\begin{array}{c}
m \\
2
\end{array}\right)\left(\begin{array}{c}
n-m \\
2
\end{array}\right) \\
= & \lambda m\left[\left(\begin{array}{c}
n-1 \\
3
\end{array}\right)-\left(\begin{array}{c}
m-1 \\
3
\end{array}\right)\right]-3 \lambda(n-m)\left(\begin{array}{c}
m \\
3
\end{array}\right)-2 \lambda\left(\begin{array}{c}
m \\
2
\end{array}\right)\left(\begin{array}{c}
n-m \\
2
\end{array}\right) \\
= & \lambda m\left(\begin{array}{c}
n-m \\
3
\end{array}\right)=\operatorname{mult}\left(u v^{3}\right) .
\end{aligned}
$$

Now, we claim that we can color the $v^{4}$-edges such that

$$
\operatorname{mult}_{j}\left(v^{4}\right)=\operatorname{mult}_{j}\left(u^{2} v^{2}\right)-\iota_{i j} \quad j \in \kappa_{i}, i=1,2 .
$$

By $(9), \operatorname{mult}_{j}\left(u^{2} v^{2}\right) \geqslant \iota_{1 j}$ for $j \in \kappa_{1}$, and mult $_{j}\left(u^{2} v^{2}\right) \geqslant \iota_{2 j}$ for $j \in \kappa_{2}$. Therefore, $\operatorname{mult}_{j}\left(v^{4}\right) \geqslant 0$ for $j \in \kappa$. Moreover, $\iota_{i j} \in \mathbb{Z}$ for $j \in \kappa_{i}, i=1,2$, and so $\operatorname{mult}_{j}\left(v^{4}\right) \in \mathbb{Z}$ for $j \in \kappa$. The following confirms that the coloring of the $v^{4}$-edges satisfying (11) is possible.

$$
\begin{aligned}
\sum_{j \in \kappa} \operatorname{mult}_{j}\left(v^{4}\right)= & \sum_{j \in \kappa_{1}} \operatorname{mult}_{j}\left(v^{4}\right)+\sum_{j \in \kappa_{2}} \operatorname{mult}_{j}\left(v^{4}\right) \\
= & q\left(\frac{s n}{4}-s m+\frac{3 r m}{4}\right)+(k-q)\left(\frac{s n}{4}-s m\right) \\
& \quad+2 \sum_{j \in \kappa} \operatorname{mult}_{j}\left(u^{3} v\right)+\sum_{j \in \kappa} \operatorname{mult}_{j}\left(u^{2} v^{2}\right) \\
& =\frac{k s n}{4}-k s m+\frac{3 q r m}{4}+2 \lambda(n-m)\left(\begin{array}{c}
m \\
3
\end{array}\right)+\lambda\left(\begin{array}{c}
m \\
2
\end{array}\right)\left(\begin{array}{c}
n-m \\
2
\end{array}\right) \\
& =\lambda\left(\begin{array}{c}
n \\
4
\end{array}\right)-\lambda m\left(\begin{array}{c}
n-1 \\
3
\end{array}\right)+3 \lambda\left(\begin{array}{c}
m \\
4
\end{array}\right)+2 \lambda(n-m)\left(\begin{array}{c}
m \\
3
\end{array}\right)+\lambda\left(\begin{array}{c}
m \\
2
\end{array}\right)\left(\begin{array}{c}
n-m \\
2
\end{array}\right) \\
& =\lambda\left(\begin{array}{c}
n \\
4
\end{array}\right)-\lambda m\left(\begin{array}{c}
n-1 \\
3
\end{array}\right)+3 \lambda\left(\begin{array}{c}
m \\
4
\end{array}\right)+\lambda m\left[\left(\begin{array}{c}
n-1 \\
3
\end{array}\right)-\left(\begin{array}{c}
m-1 \\
3
\end{array}\right)\right]-\lambda\left(\begin{array}{c}
n \\
4
\end{array}\right) \\
& +\lambda\left(\begin{array}{c}
m \\
4
\end{array}\right)+\lambda\left(\begin{array}{c}
n-m \\
4
\end{array}\right) \\
& =\lambda\left(\begin{array}{c}
n-m \\
4
\end{array}\right)=\operatorname{mult}\left(v^{4}\right) .
\end{aligned}
$$


Finally, we prove that our coloring satisfies (8). For $j \in \kappa$,

$$
\begin{aligned}
& \operatorname{deg}_{j}(x)= \begin{cases}3 \operatorname{mult}_{j}\left(u^{3} v\right)+2 \operatorname{mult}_{j}\left(u^{2} v^{2}\right)+\operatorname{mult}_{j}\left(u v^{3}\right) & \text { if } x=u \\
\operatorname{mult}_{j}\left(u^{3} v\right)+2 \operatorname{mult}_{j}\left(u^{2} v^{2}\right)+3 \operatorname{mult}_{j}\left(u v^{3}\right)+4 \operatorname{mult}_{j}\left(v^{4}\right) & \text { if } x=v\end{cases} \\
& = \begin{cases}3 \operatorname{mult}_{j}\left(u^{3} v\right)+2 \rho_{i j} & \text { if } x=u, j \in \kappa_{i}, i=1,2 \\
\operatorname{mult}_{j}\left(u^{3} v\right)+6 \rho_{i j}-4 \iota_{i j} & \text { if } x=v, j \in \kappa_{i}, i=1,2\end{cases} \\
& = \begin{cases} \begin{cases}m(s-r) & \text { for } j \in \kappa_{1} \\
s m & \text { for } j \in \kappa_{2}\end{cases} & \text { if } x=u \\
s(n-m) & \text { if } x=v .\end{cases}
\end{aligned}
$$

\section{Colorings II}

In this section we show that we can color the $u^{3} v$-edges and $u^{2} v^{2}$-edges of $\mathcal{F}$ such that (9) holds. Since we will frequently deal with $u^{3} v$-edges and $u^{2} v^{2}$-edges, let us make the following abbreviation.

$$
\begin{cases}e:=\lambda(n-m)\left(\begin{array}{c}
m \\
3
\end{array}\right), & \\
f:=\lambda\left(\begin{array}{c}
m \\
2
\end{array}\right)\left(\begin{array}{c}
n-m \\
2
\end{array}\right), & \\
e_{j}:=\operatorname{mult}_{j}\left(u^{3} v\right), & \text { for } j \in \kappa, \\
f_{j}:=\operatorname{mult}_{j}\left(u^{2} v^{2}\right), & \text { for } j \in \kappa .\end{cases}
$$

The following simple lemma will be quite useful.

Lemma 5.1. Let $c, k \in \mathbb{N}$ and $S=\{1, \ldots, k\}$. Let $a_{i} \in \mathbb{Z}, b_{i} \in \mathbb{R}$ with $b_{i} \geqslant 0, a_{i} \leqslant b_{i}$ for $i \in S$. Moreover, suppose that $a_{i} \geqslant 0$ for $i \in I \subseteq S$, and $a_{i}<0$ for $i \in S \backslash I$. Consider the following system.

$$
\begin{cases}\sum_{i \in \kappa} x_{i}=c, & \\ a_{i} \leqslant x_{i} \leqslant b_{i}, & i \in S, \\ x_{i} \in \mathbb{N} \cup\{0\}, & i \in S .\end{cases}
$$

Then (12) has a solution if and only if

$$
\sum_{i \in I} a_{i} \leqslant c \leqslant \sum_{i \in S}\left\lfloor b_{i}\right\rfloor
$$

Proof. First, suppose that (12) has a solution $\left(x_{1}^{*}, \ldots, x_{k}^{*}\right)$. Since $a_{i} \leqslant x_{i}^{*} \leqslant b_{i}$ for $i \in S$ and $x_{i}$ s are non-negative integers, we have $a_{i} \leqslant x_{i}^{*} \leqslant\left\lfloor b_{i}\right\rfloor$ for $i \in I$, and $0 \leqslant x_{i}^{*} \leqslant\left\lfloor b_{i}\right\rfloor$ for $i \in S \backslash I$. Combining this with $\sum_{i \in S} x_{i}^{*}=c$ we have

$$
\sum_{i \in I} a_{i} \leqslant c \leqslant \sum_{i \in S}\left\lfloor b_{i}\right\rfloor
$$

Conversely, suppose that $\sum_{i \in I} a_{i} \leqslant c \leqslant \sum_{i \in S}\left\lfloor b_{i}\right\rfloor$. Let $x_{i}^{*}=a_{i}$ for $i \in I$, and $x_{i}^{*}=0$ for $i \in S \backslash I$. Clearly, (i) $x_{i}^{*} \in \mathbb{N} \cup\{0\}$ for $i \in S$. Since by our hypothesis, $a_{i} \leqslant b_{i}$ for $i \in S$, we have that (ii) $a_{i} \leqslant x_{i}^{*} \leqslant b_{i}$ for $i \in S$. At this point, $\sum_{i \in S} x_{i}^{*}=\sum_{i \in I} a_{i} \leqslant c$, but since 
$c \leqslant \sum_{i \in S}\left\lfloor b_{i}\right\rfloor$, we can increase the value of $x_{i}^{*} \mathrm{~s}$ without violating conditions (i) and (ii) so that their sum is exactly $c$.

By Lemma 4.1, if we show that the following system has a solution, then we are done.

$$
\left\{\begin{array}{l}
\sum_{j \in \kappa} e_{j}=e, \\
\sum_{j \in \kappa} f_{j}=f, \\
\iota_{1} \leqslant e_{j} \leqslant \rho_{1}, \quad j \in \kappa_{1}, \\
\iota_{2} \leqslant e_{j} \leqslant \rho_{2}, \quad j \in \kappa_{2}, \\
\iota_{1 j} \leqslant f_{j} \leqslant \rho_{1 j}, \quad j \in \kappa_{1}, \\
\iota_{2 j} \leqslant f_{j} \leqslant \rho_{2 j}, \quad j \in \kappa_{2} .
\end{array}\right.
$$

By Lemma 5.1, this system has a solution if and only if

$$
\begin{gathered}
\sum_{j \in \kappa_{1}, \iota_{1} \geqslant 0} \iota_{1}+\sum_{j \in \kappa_{2}, \iota_{2} \geqslant 0} \iota_{2} \leqslant e \leqslant q\left\lfloor\rho_{1}\right\rfloor+(k-q)\left\lfloor\rho_{2}\right\rfloor, \\
\sum_{j \in J_{1}} \iota_{1 j}+\sum_{j \in J_{2}} \iota_{2 j} \leqslant f \leqslant \sum_{j \in \kappa_{1}}\left\lfloor\rho_{1 j}\right\rfloor+\sum_{j \in \kappa_{2}}\left\lfloor\rho_{2 j}\right\rfloor,
\end{gathered}
$$

where for $i=1,2$,

$$
J_{i}:=\left\{j \in \kappa_{i} \mid \iota_{i j} \geqslant 0\right\}=\left\{j \in \kappa_{i} \mid e_{j} \leqslant \rho_{i}^{\prime}\right\} .
$$

Since $\iota_{2}>\iota_{1}$, either $\iota_{2}>\iota_{1} \geqslant 0$, or $\iota_{2} \geqslant 0 \geqslant \iota_{1}$, or $0 \geqslant \iota_{2}>\iota_{1}$. Thus,

$$
\sum_{j \in \kappa_{1}, \iota_{1} \geqslant 0} \iota_{1}+\sum_{j \in \kappa_{2}, \iota_{2} \geqslant 0} \iota_{2}= \begin{cases}(k-q) \iota_{2}+q \iota_{1} & \text { if } \iota_{2}>\iota_{1} \geqslant 0, \\ (k-q) \iota_{2} & \text { if } \iota_{2} \geqslant 0 \geqslant \iota_{1}, \\ 0 & \text { if } 0 \geqslant \iota_{2}>\iota_{1} .\end{cases}
$$

But by Lemma 6.1 and (N6)

$$
e \geqslant \begin{cases}(k-q) \iota_{2}+q \iota_{1} & \text { if } \iota_{2}>\iota_{1} \geqslant 0 \\ (k-q) \iota_{2} & \text { if } \iota_{2} \geqslant 0 \geqslant \iota_{1} \\ 0 & \text { if } 0 \geqslant \iota_{2}>\iota_{1}\end{cases}
$$

Again, by Lemma 6.1,

$$
e \leqslant q\left\lfloor\rho_{1}\right\rfloor+(k-q)\left\lfloor\rho_{2}\right\rfloor
$$

So that settles (14). Unfortunately, (15) depends on (14), so we cannot necessarily go on to show that (15) is satisfied. The following example illustrates this issue.

Example 5.2. Let $m=6, r=2, n=9, s=4, \lambda=1$. It is easy to verify that conditions (1)-(4) of Theorem 1.1 are satisfied, and that $q=5, k=14, e=60, f=45, \iota_{1}=0, \rho_{1}=$ $4, \iota_{2}=6, \rho_{2}=8$. Clearly, $e_{1}=4, e_{2}=e_{3}=e_{4}=e_{5}=0, e_{6}=8, e_{7}=e_{8}=e_{9}=e_{10}=e_{11}=$ $e_{12}=e_{13}=e_{14}=6$ is a solution to

$$
\begin{cases}\sum_{j=1}^{14} e_{j}=60, & \\ 0 \leqslant e_{j} \leqslant 4, & j=1, \ldots, 5 \\ 6 \leqslant e_{j} \leqslant 8, & j=6, \ldots, 14\end{cases}
$$


For $j=1, \ldots, 5, \iota_{1 j}=6-2 e_{j}, \rho_{1 j}=6-3 e_{j} / 2$, and for $j=6, \ldots, 14, \iota_{2 j}=15-2 e_{j}, \rho_{2 j}=$ $12-3 e_{j} / 2$. Therefore, $\iota_{11}=-2, \rho_{11}=0$, and $\iota_{1 j}=\rho_{1 j}=6$ for $j=2, \ldots, 5$. Moreover, $\iota_{26}=-1, \rho_{26}=0$, and $\iota_{2 j}=\rho_{2 j}=3$ for $j=7, \ldots, 14$. The following system has no solution.

$$
\left\{\begin{aligned}
\sum_{j=1}^{14} f_{j} & =45 \\
-2 & \leqslant f_{1} \leqslant 0 \\
6 & \leqslant f_{j} \leqslant 6, \quad j=2, \ldots, 5 \\
-1 & \leqslant f_{6} \leqslant 0, \\
3 & \leqslant f_{j} \leqslant 3, \quad j=7, \ldots, 14 .
\end{aligned}\right.
$$

This is because only one choice of $f_{i}$ s, namely $f_{1}=0, f_{2}=f_{3}=f_{4}=f_{5}=6, f_{6}=0, f_{7}=$ $f_{8}=f_{9}=f_{10}=f_{11}=f_{12}=f_{13}=f_{14}=3$, satisfies the inequalities in the system, but $\sum_{j=1}^{14} f_{j}=48 \neq 45$.

Since $\rho_{2}^{\prime}>\rho_{1}^{\prime}$, either $\rho_{2}^{\prime}>\rho_{1}^{\prime} \geqslant 0$, or $\rho_{2}^{\prime} \geqslant 0 \geqslant \rho_{1}^{\prime}$, or $0 \geqslant \rho_{2}^{\prime}>\rho_{1}^{\prime}$. To solve (13), there are six cases to consider (see Table 2 ).

5.1. $\iota_{1}<\iota_{2} \leqslant \mathbf{0}, \rho_{1}^{\prime}<\rho_{2}^{\prime}<\mathbf{0}$. First, we color the $u^{3} v$-edges so that

$$
\begin{cases}\iota_{1} \leqslant \rho_{1}^{\prime}<0 \leqslant e_{j} \leqslant \rho_{1} & \text { if } j \in \kappa_{1}, \\ \iota_{2} \leqslant \rho_{2}^{\prime}<0 \leqslant e_{j} \leqslant \rho_{2} & \text { if } j \in \kappa_{2} .\end{cases}
$$

By Lemma 6.1, $e \leqslant q\left\lfloor\rho_{1}\right\rfloor+(k-q)\left\lfloor\rho_{2}\right\rfloor$, so this is possible. Then, we color the $u^{2} v^{2}$-edges so that

$$
\begin{cases}\iota_{1 j} \leqslant 0 \leqslant f_{j} \leqslant \rho_{1 j} & \text { if } j \in \kappa_{1} \\ \iota_{2 j} \leqslant 0 \leqslant f_{j} \leqslant \rho_{2 j} & \text { if } j \in \kappa_{2}\end{cases}
$$

By Lemma $6.5, f \leqslant \sum_{j \in \kappa_{1}}\left\lfloor\rho_{1 j}\right\rfloor+\sum_{j \in \kappa_{2}}\left\lfloor\rho_{2 j}\right\rfloor$, so this is also possible.

5.2. $0 \leqslant \iota_{1}<\iota_{2}, 0 \leqslant \rho_{1}^{\prime}<\rho_{2}^{\prime}$. First, we color the $u^{3} v$-edges so that

$$
\begin{cases}0 \leqslant \iota_{1} \leqslant e_{j} \leqslant \rho_{1}^{\prime} \leqslant \rho_{1} & \text { if } j \in \kappa_{1}, \\ 0 \leqslant \iota_{2} \leqslant e_{j} \leqslant \rho_{2}^{\prime} \leqslant \rho_{2} & \text { if } j \in \kappa_{2} .\end{cases}
$$

By Lemmas 6.1 and 6.3, $q \iota_{1}+(k-q) \iota_{2} \leqslant e \leqslant q\left\lfloor\rho_{1}^{\prime}\right\rfloor+(k-q)\left\lfloor\rho_{2}^{\prime}\right\rfloor$, so this is possible. Then, we color the $u^{2} v^{2}$-edges so that

$$
\begin{cases}0 \leqslant \iota_{1 j} \leqslant f_{j} \leqslant \rho_{1 j} & \text { if } j \in \kappa_{1}, \\ 0 \leqslant \iota_{2 j} \leqslant f_{j} \leqslant \rho_{2 j} & \text { if } j \in \kappa_{2} .\end{cases}
$$

By Lemmas 6.4 and 6.5, $\sum_{j \in \kappa_{1}} \iota_{1 j}+\sum_{j \in \kappa_{2}} \iota_{2 j} \leqslant f \leqslant \sum_{j \in \kappa_{1}}\left\lfloor\rho_{1 j}\right\rfloor+\sum_{j \in \kappa_{2}}\left\lfloor\rho_{2 j}\right\rfloor$, so this is also possible.

5.3. $\iota_{\mathbf{1}}<\mathbf{0} \leqslant \boldsymbol{\iota}_{\mathbf{2}}, \mathbf{0} \leqslant \boldsymbol{\rho}_{\mathbf{1}}^{\prime}<\boldsymbol{\rho}_{\mathbf{2}}^{\prime}$. First, we color the $u^{3} v$-edges so that

$$
\begin{cases}\iota_{1}<0 \leqslant e_{j} \leqslant \rho_{1}^{\prime} \leqslant \rho_{1} & \text { if } j \in \kappa_{1}, \\ 0 \leqslant \iota_{2} \leqslant e_{j} \leqslant \rho_{2}^{\prime} \leqslant \rho_{2} & \text { if } j \in \kappa_{2} .\end{cases}
$$

By (N6) and Lemma 6.3, $(k-q) \iota_{2} \leqslant e \leqslant q\left\lfloor\rho_{1}^{\prime}\right\rfloor+(k-q)\left\lfloor\rho_{2}^{\prime}\right\rfloor$, so this is possible. Then, we color the $u^{2} v^{2}$-edges so that

$$
\begin{cases}0 \leqslant \iota_{1 j} \leqslant f_{j} \leqslant \rho_{1 j} & \text { if } j \in \kappa_{1} \\ 0 \leqslant \iota_{2 j} \leqslant f_{j} \leqslant \rho_{2 j} & \text { if } j \in \kappa_{2}\end{cases}
$$




\begin{tabular}{|c|c|}
\hline $\begin{array}{ll}\iota_{1}=s m-\frac{s n}{2}-\frac{r m}{2} & \\
\iota_{2}=s m-\frac{s n}{2} & \text { if } \kappa_{2} \neq \varnothing \\
\rho_{1}=\frac{s m}{3}-\frac{r m}{3} & \\
\rho_{2}=\frac{s m}{3} & \text { if } \kappa_{2} \neq \varnothing \\
\rho_{1}^{\prime}=\frac{s m}{2}-\frac{s n}{8}-\frac{3 r m}{8} & \text { if } \kappa_{2} \neq \varnothing \\
\rho_{2}^{\prime}=\frac{s m}{2}-\frac{s n}{8} & \text { if } j \in \kappa_{1} \\
\iota_{1 j}=s m-\frac{s n}{4}-2 \operatorname{mult}_{j}\left(u^{3} v\right)-\frac{3 r m}{4} & \text { if } j \in \kappa_{2} \\
\iota_{2 j}=s m-\frac{s n}{4}-2 \operatorname{mult}_{j}\left(u^{3} v\right) & \text { if } j \in \kappa_{1} \\
\rho_{1 j}=\frac{s m}{2}-\frac{3}{2} \operatorname{mult}_{j}\left(u^{3} v\right)-\frac{r m}{2} & \text { if } j \in \kappa_{2} \\
\rho_{2 j}=\frac{s m}{2}-\frac{3}{2} \operatorname{mult}_{j}\left(u^{3} v\right) & \end{array}$ & $\begin{array}{l}\iota_{1}, \iota_{2}, \iota_{1 j}, \iota_{2 j} \in \mathbb{Z} \\
\rho_{1}, \rho_{2}, \rho_{1}^{\prime}, \rho_{2}^{\prime}, \rho_{1 j}, \rho_{2 j} \in \mathbb{R}\end{array}$ \\
\hline $\begin{array}{l}\iota_{2}>\iota_{1} \\
\rho_{2}>\rho_{1} \geqslant 0 \\
\rho_{2}^{\prime}>\rho_{1}^{\prime} \\
\iota_{1} \leqslant \rho_{1}^{\prime} \leqslant \rho_{1} \\
\iota_{2} \leqslant \rho_{2}^{\prime} \leqslant \rho_{2} \quad \text { if } \kappa_{2} \neq \varnothing\end{array}$ & $\begin{array}{ll}\rho_{1}=0, \rho_{1}^{\prime}<0 & \text { if } r=s \\
\iota_{1 j}<0, \rho_{1 j}=0 & \text { if } r=s\end{array}$ \\
\hline $\begin{array}{l}\iota_{1} \geqslant 0 \Longleftrightarrow n \leqslant\left(2-\frac{r}{s}\right) m \\
\iota_{2} \geqslant 0 \Longleftrightarrow n \leqslant 2 m \\
\rho_{1}^{\prime} \geqslant 0 \Longleftrightarrow n \leqslant\left(4-\frac{3 r}{s}\right) m \\
\rho_{2}^{\prime} \geqslant 0 \Longleftrightarrow n \leqslant 4 m\end{array}$ & $\begin{array}{ll}\iota_{1 j} \geqslant 0 \Longleftrightarrow \operatorname{mult}_{j}\left(u^{3} v\right) \leqslant \rho_{1}^{\prime} & \text { if } j \in \kappa_{1} \\
\iota_{2 j} \geqslant 0 \Longleftrightarrow \operatorname{mult}_{j}\left(u^{3} v\right) \leqslant \rho_{2}^{\prime} & \text { if } j \in \kappa_{2} \\
\rho_{1 j} \geqslant 0 \Longleftrightarrow \operatorname{mult}_{j}\left(u^{3} v\right) \leqslant \rho_{1} & \text { if } j \in \kappa_{1} \\
\rho_{2 j} \geqslant 0 \Longleftrightarrow \operatorname{mult}_{j}\left(u^{3} v\right) \leqslant \rho_{2} & \text { if } j \in \kappa_{2} \\
\rho_{1 j} \geqslant \iota_{1 j} \Longleftrightarrow \operatorname{mult}_{j}\left(u^{3} v\right) \geqslant \iota_{1} & \text { if } j \in \kappa_{1} \\
\rho_{2 j} \geqslant \iota_{2 j} \Longleftrightarrow \operatorname{mult}_{j}\left(u^{3} v\right) \geqslant \iota_{2} & \text { if } j \in \kappa_{1}\end{array}$ \\
\hline
\end{tabular}

TABLE 1.

By Lemmas 6.4 and 6.5, $\sum_{j \in \kappa_{1}} \iota_{1 j}+\sum_{j \in \kappa_{2}} \iota_{2 j} \leqslant f \leqslant \sum_{j \in \kappa_{1}}\left\lfloor\rho_{1 j}\right\rfloor+\sum_{j \in \kappa_{2}}\left\lfloor\rho_{2 j}\right\rfloor$, so this is also possible. 


\begin{tabular}{|l|l|}
\hline$\iota_{1}<\iota_{2} \leqslant 0, \rho_{1}^{\prime}<\rho_{2}^{\prime}<0$ & $n>4 m$ \\
\hline $0 \leqslant \iota_{1}<\iota_{2}, 0 \leqslant \rho_{1}^{\prime}<\rho_{2}^{\prime}$ & $n \leqslant(2-r / s) m$ \\
\hline$\iota_{1}<0 \leqslant \iota_{2}, 0 \leqslant \rho_{1}^{\prime}<\rho_{2}^{\prime}$ & $(2-r / s) m<n \leqslant \min \{2 m,(4-3 r / s) m\}$ \\
\hline$\iota_{1}<0<\iota_{2}, \rho_{1}^{\prime}<0 \leqslant \rho_{2}^{\prime}$ & $(4-3 r / s) m<n<2 m, s<3 r / 2$ \\
\hline$\iota_{1}<\iota_{2}<0,0 \leqslant \rho_{1}^{\prime}<\rho_{2}^{\prime}$ & $2 m<n \leqslant(4-3 r / s) m, s>3 r / 2$ \\
\hline$\iota_{1}<\iota_{2} \leqslant 0, \rho_{1}^{\prime}<0 \leqslant \rho_{2}^{\prime}$ & $\max \{2 m,(4-3 r / s) m\}<n \leqslant 4 m$ \\
\hline
\end{tabular}

TABLE 2.

5.4. $\iota_{\mathbf{1}}<0<\iota_{\mathbf{2}}, \boldsymbol{\rho}_{\mathbf{1}}^{\prime}<0 \leqslant \boldsymbol{\rho}_{\mathbf{2}}^{\prime}$. First, we color the $u^{3} v$-edges so that

$$
\left\{\begin{aligned}
\iota_{1} \leqslant \rho_{1}^{\prime}<0=e_{j} \leqslant \rho_{1} & \text { if } j \in \kappa_{1}, \\
0<\iota_{2} \leqslant e_{j} \leqslant \rho_{2}^{\prime} \leqslant \rho_{2} & \text { if } j \in \kappa_{2} .
\end{aligned}\right.
$$

By (N6) and Lemma 6.3, $(k-q) \iota_{2} \leqslant e \leqslant(k-q)\left\lfloor\rho_{2}^{\prime}\right\rfloor$, so this is possible. Then, we color the $u^{2} v^{2}$-edges so that

$$
\begin{cases}\iota_{1 j} \leqslant 0 \leqslant f_{j} \leqslant \rho_{1 j} & \text { if } j \in \kappa_{1}, \\ 0 \leqslant \iota_{2 j} \leqslant f_{j} \leqslant \rho_{2 j} & \text { if } j \in \kappa_{2} .\end{cases}
$$

By Lemmas 6.4 and $6.5, \sum_{j \in \kappa_{2}} \iota_{2 j} \leqslant f \leqslant \sum_{j \in \kappa_{1}}\left\lfloor\rho_{1 j}\right\rfloor+\sum_{j \in \kappa_{2}}\left\lfloor\rho_{2 j}\right\rfloor$, so this is also possible.

5.5. $\iota_{\mathbf{1}}<\boldsymbol{\iota}_{\mathbf{2}}<\mathbf{0}, \mathbf{0} \leqslant \boldsymbol{\rho}_{\mathbf{1}}^{\prime}<\boldsymbol{\rho}_{\mathbf{2}}^{\prime}$. First, we show that for $i=1,2,\left\lceil\rho_{i}^{\prime}\right\rceil \leqslant \rho_{i}$. Since $(m, r, \lambda)$ and $(n, s, \lambda)$ are admissible, we have $\left\lceil\rho_{i}^{\prime}\right\rceil \leqslant \rho_{i}^{\prime}+1 / 2$ for $i=1,2$. Moreover, $m \geqslant 4, s \geqslant\lceil 3 r / 2\rceil \geqslant 2$, and $n \geqslant 2 m$. Therefore,

$$
\begin{aligned}
24\left(\rho_{i}-\left\lceil\rho_{i}^{\prime}\right]\right) & \geqslant 24\left(\rho_{i}-\rho_{i}^{\prime}-1 / 2\right) \\
& = \begin{cases}3 s n-4 s m+r m-12 & \text { if } i=1, \\
3 s n-4 s m-12 & \text { if } i=2 .\end{cases} \\
& \geqslant 3 s n-4 s m-12 \\
& \geqslant 2 s m-12>0 .
\end{aligned}
$$

There are three cases to consider.

(i) $e \leqslant q\left\lfloor\rho_{1}^{\prime}\right\rfloor+(k-q)\left\lfloor\rho_{2}^{\prime}\right\rfloor$ : First, we color the $u^{3} v$-edges so that

$$
\begin{cases}\iota_{1}<0 \leqslant e_{j} \leqslant \rho_{1}^{\prime} \leqslant \rho_{1} & \text { if } j \in \kappa_{1} \\ \iota_{2}<0 \leqslant e_{j} \leqslant \rho_{2}^{\prime} \leqslant \rho_{2} & \text { if } j \in \kappa_{2} .\end{cases}
$$

By our hypothesis, $e \leqslant q\left\lfloor\rho_{1}^{\prime}\right\rfloor+(k-q)\left\lfloor\rho_{2}^{\prime}\right\rfloor$, so this is possible. Then, we color the $u^{2} v^{2}$-edges so that

$$
\begin{cases}0 \leqslant \iota_{1 j} \leqslant f_{j} \leqslant \rho_{1 j} & \text { if } j \in \kappa_{1}, \\ 0 \leqslant \iota_{2 j} \leqslant f_{j} \leqslant \rho_{2 j} & \text { if } j \in \kappa_{2} .\end{cases}
$$

By Lemmas 6.4 and 6.5, $\sum_{j \in \kappa_{1}} \iota_{1 j}+\sum_{j \in \kappa_{2}} \iota_{2 j} \leqslant f \leqslant \sum_{j \in \kappa_{1}}\left\lfloor\rho_{1 j}\right\rfloor+\sum_{j \in \kappa_{2}}\left\lfloor\rho_{2 j}\right\rfloor$, so this is also possible. 
(ii) $e \geqslant q\left\lceil\rho_{1}^{\prime}\right\rceil+(k-q)\left\lceil\rho_{2}^{\prime}\right\rceil:$ First, we color the $u^{3} v$-edges so that

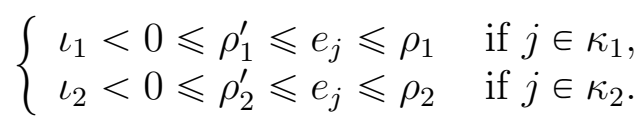

By our hypothesis and Lemma 6.1, $q\left\lceil\rho_{1}^{\prime}\right\rceil+(k-q)\left\lceil\rho_{2}^{\prime}\right\rceil \leqslant e \leqslant q\left\lfloor\rho_{1}\right\rfloor+(k-q)\left\lfloor\rho_{2}\right\rfloor$, so this is possible. Then, we color the $u^{2} v^{2}$-edges so that

$$
\left\{\begin{array}{l}
\iota_{1 j} \leqslant 0 \leqslant f_{j} \leqslant \rho_{1 j} \quad \text { if } j \in \kappa_{1}, \\
\iota_{2 j} \leqslant 0 \leqslant f_{j} \leqslant \rho_{2 j} \quad \text { if } j \in \kappa_{2} .
\end{array}\right.
$$

By Lemma $6.5, f \leqslant \sum_{j \in \kappa_{1}}\left\lfloor\rho_{1 j}\right\rfloor+\sum_{j \in \kappa_{2}}\left\lfloor\rho_{2 j}\right\rfloor$, so this is also possible.

(iii) $q\left\lfloor\rho_{1}^{\prime}\right\rfloor+(k-q)\left\lfloor\rho_{2}^{\prime}\right\rfloor<e<q\left\lceil\rho_{1}^{\prime}\right\rceil+(k-q)\left\lceil\rho_{2}^{\prime}\right\rceil$ : Observe that at most one of $\rho_{1}^{\prime}$ and $\rho_{2}^{\prime}$ is an integer. We color the $u^{3} v$-edges so that

$$
\begin{cases}\iota_{1}<0 \leqslant\left\lfloor\rho_{1}^{\prime}\right\rfloor \leqslant e_{j} \leqslant\left\lceil\rho_{1}^{\prime}\right] \leqslant \rho_{1} & \text { if } j \in \kappa_{1}, \\ \iota_{2}<0 \leqslant\left\lfloor\rho_{2}^{\prime}\right\rfloor \leqslant e_{j} \leqslant\left\lceil\rho_{2}^{\prime}\right\rceil \leqslant \rho_{2} & \text { if } j \in \kappa_{2} .\end{cases}
$$

By our hypothesis, $q\left\lfloor\rho_{1}^{\prime}\right\rfloor+(k-q)\left\lfloor\rho_{2}^{\prime}\right\rfloor<e<q\left\lceil\rho_{1}^{\prime}\right\rceil+(k-q)\left\lceil\rho_{2}^{\prime}\right\rceil$, so this is possible. Since

$$
\iota_{i j}=2\left(\rho_{i}^{\prime}-e_{j}\right) \in\{-1,0,1\} \text { for } i=1,2, j \in \kappa_{i},
$$

we have

$$
\sum_{j \in J_{1}} \iota_{1 j}+\sum_{j \in J_{2}} \iota_{2 j} \leqslant k
$$

Now, we color the $u^{2} v^{2}$-edges so that

$$
\begin{cases}\iota_{1 j} \leqslant f_{j} \leqslant \rho_{1 j} & \text { if } j \in \kappa_{1}, \\ \iota_{2 j} \leqslant f_{j} \leqslant \rho_{2 j} & \text { if } j \in \kappa_{2} .\end{cases}
$$

By (16), and Lemmas 6.5 and 6.6,

$$
\sum_{j \in J_{1}} \iota_{1 j}+\sum_{j \in J_{2}} \iota_{2 j} \leqslant k \leqslant f \leqslant \sum_{j \in \kappa_{1}}\left\lfloor\rho_{1 j}\right\rfloor+\sum_{j \in \kappa_{2}}\left\lfloor\rho_{2 j}\right\rfloor,
$$

so coloring the $u^{2} v^{2}$-edges is also possible.

5.6. $\iota_{1}<\iota_{2} \leqslant 0, \rho_{1}^{\prime}<0 \leqslant \rho_{2}^{\prime}$. First, we show that $\left[\rho_{2}^{\prime}\right] \leqslant \rho_{2}$. We have

$$
z:=24\left(\rho_{2}-\left\lceil\rho_{2}^{\prime}\right\rceil\right)=24\left(\rho_{2}-\rho_{2}^{\prime}-1 / 2\right)=3 s n-4 s m-12
$$

Since $n \geqslant \max \{2 m,(4-3 r / s) m\}$, we have

$$
n \geqslant \begin{cases}2 m & \text { if } s<3 r / 2, \\ (4-3 r / s) m & \text { if } s \geqslant 3 r / 2 .\end{cases}
$$

Recall that if $m=4$, then $r \geqslant 2$. If $m=5$, then since $(m, r, \lambda)$ is admissible, we have $r \geqslant 4$. Therefore, if $n \geqslant 2 m$, then $z \geqslant 2 s m-12 \geqslant 0$. If $n \geqslant(4-3 r / s) m$ and $s \geqslant 3 r / 2$, then

$$
\begin{aligned}
z & \geqslant 3 s((4-3 r / s) m)-4 s m-12 \\
& =m(8 s-9 r)-12 \\
& \geqslant m(12 r-9 r)-12 \\
& \geqslant 3 r m-12 \geqslant 0 .
\end{aligned}
$$

There are three cases to consider. 
(i) $e \leqslant(k-q)\left\lfloor\rho_{2}^{\prime}\right\rfloor$ : First, we color the $u^{3} v$-edges so that

$$
\left\{\begin{aligned}
\iota_{1} \leqslant \rho_{1}^{\prime}<0 & =e_{j} \leqslant \rho_{1} & & \text { if } j \in \kappa_{1}, \\
\iota_{2} & \leqslant 0 \leqslant e_{j} \leqslant \rho_{2}^{\prime} \leqslant \rho_{2} & & \text { if } j \in \kappa_{2} .
\end{aligned}\right.
$$

This is possible by our hypothesis. Then, we color the $u^{2} v^{2}$-edges so that

$$
\left\{\begin{array}{l}
\iota_{1 j} \leqslant 0 \leqslant f_{j} \leqslant \rho_{1 j} \\
0 \leqslant \iota_{2 j} \leqslant f_{j} \leqslant \rho_{2 j} \quad \text { if } j \in \kappa_{1}, \\
j \in \kappa_{2} .
\end{array}\right.
$$

By Lemmas 6.4 and $6.5, \sum_{j \in \kappa_{2}} \iota_{2 j} \leqslant f \leqslant \sum_{j \in \kappa_{1}}\left\lfloor\rho_{1 j}\right\rfloor+\sum_{j \in \kappa_{2}}\left\lfloor\rho_{2 j}\right\rfloor$, so this is also possible.

(ii) $e \geqslant(k-q)\left[\rho_{2}^{\prime}\right]$ : We color the $u^{3} v$-edges such that

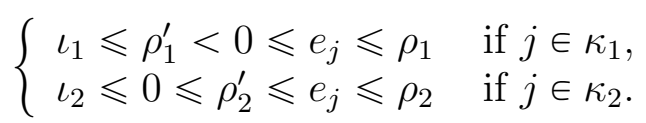

By our hypothesis and Lemma 6.1, $(k-q)\left\lceil\rho_{2}^{\prime}\right\rceil \leqslant e \leqslant q\left\lfloor\rho_{1}\right\rfloor+(k-q)\left\lfloor\rho_{2}\right\rfloor$, so this is possible. Then, we color the $u^{2} v^{2}$-edges so that

$$
\begin{cases}\iota_{1 j} \leqslant 0 \leqslant f_{j} \leqslant \rho_{1 j} & \text { if } j \in \kappa_{1}, \\ \iota_{2 j} \leqslant 0 \leqslant f_{j} \leqslant \rho_{2 j} & \text { if } j \in \kappa_{2} .\end{cases}
$$

By Lemma 6.5, $f \leqslant \sum_{j \in \kappa_{1}}\left\lfloor\rho_{1 j}\right\rfloor+\sum_{j \in \kappa_{2}}\left\lfloor\rho_{2 j}\right\rfloor$, so this is also possible.

(iii) $(k-q)\left\lfloor\rho_{2}^{\prime}\right\rfloor<e<(k-q)\left\lceil\rho_{2}^{\prime}\right\rfloor$ : Observe that $\rho_{2}^{\prime} \notin \mathbb{N}$. We color the $u^{3} v$-edges such that

$$
\left\{\begin{array}{cl}
\iota_{1} \leqslant \rho_{1}^{\prime}<0=e_{j} \leqslant \rho_{1} & \text { if } j \in \kappa_{1}, \\
\iota_{2} \leqslant 0 \leqslant\left\lfloor\rho_{2}^{\prime}\right\rfloor \leqslant e_{j} \leqslant\left\lceil\rho_{2}^{\prime}\right\rceil \leqslant \rho_{2} & \text { if } j \in \kappa_{2} .
\end{array}\right.
$$

By our hypothesis, $(k-q)\left\lfloor\rho_{2}^{\prime}\right\rfloor<e<(k-q)\left\lceil\rho_{2}^{\prime}\right\rceil$, so this is possible. Since

$$
\iota_{2 j}=2\left(\rho_{2}^{\prime}-e_{j}\right) \in\{-1,1\} \text { for } j \in \kappa_{2},
$$

we have

$$
\sum_{j \in J_{2}} \iota_{2 j} \leqslant k-q
$$

Now, we color the $u^{2} v^{2}$-edges so that

$$
\left\{\begin{aligned}
\iota_{1 j} \leqslant 0 \leqslant f_{j} \leqslant \rho_{1 j} & \text { if } j \in \kappa_{1}, \\
\iota_{2 j} \leqslant f_{j} \leqslant \rho_{2 j} & \text { if } j \in \kappa_{2} .
\end{aligned}\right.
$$

By (17), Lemmas 6.5 and 6.6,

$$
\sum_{j \in \kappa_{2}} \iota_{2 j} \leqslant k-q<k \leqslant f \leqslant \sum_{j \in \kappa_{1}}\left\lfloor\rho_{1 j}\right\rfloor+\sum_{j \in \kappa_{2}}\left\lfloor\rho_{2 j}\right\rfloor,
$$

so coloring the $u^{2} v^{2}$-edges is also possible.

\section{INEQUalities}

Before we can color the $u^{3} v$-edges and $u^{2} v^{2}$-edges of $\mathcal{F}$, we need to establish several important inequalities. Most of these multivariate inequalities have been reduced to two-variable inequalities and then have been verified by the computer algebra system Mathematica. Since a central part of our argument relies on these inequalities, we shall provide detailed proofs here. A reader is welcome to skip this section in the first reading.

For $x \in \mathbb{R}, \operatorname{frac}(x)$ denotes the fractional part of $x$ which is $x-\lfloor x\rfloor$. 


\section{Lemma 6.1.}

$$
q \iota_{1}+(k-q) \iota_{2} \leqslant e \leqslant q\left\lfloor\rho_{1}\right\rfloor+(k-q)\left\lfloor\rho_{2}\right\rfloor .
$$

Proof. The following proves the left-hand side inequality.

$$
\begin{aligned}
q \iota_{1}+(k-q) \iota_{2} & =k s m-\frac{k s n}{2}-\frac{q r m}{2} \\
& =\lambda\left(m-\frac{n}{2}\right)\left(\begin{array}{c}
n-1 \\
3
\end{array}\right)-\lambda \frac{m}{2}\left(\begin{array}{c}
m-1 \\
3
\end{array}\right) \\
& =\lambda m\left(\begin{array}{c}
n-1 \\
3
\end{array}\right)-2 \lambda\left(\begin{array}{c}
n \\
4
\end{array}\right)-2 \lambda\left(\begin{array}{c}
m \\
4
\end{array}\right) \\
& =\lambda(n-m)\left(\begin{array}{c}
m \\
3
\end{array}\right)-\frac{\lambda}{2}\left(\begin{array}{c}
n-m \\
3
\end{array}\right)(n+m-3) \\
& <\lambda(n-m)\left(\begin{array}{c}
m \\
3
\end{array}\right) .
\end{aligned}
$$

Since

$$
\begin{aligned}
3 q\left\lfloor\rho_{1}\right\rfloor+3(k-q)\left\lfloor\rho_{2}\right\rfloor & =3 q \rho_{1}+3(k-q) \rho_{2}-3 q \operatorname{frac}\left(\rho_{1}\right)-3(k-q) \operatorname{frac}\left(\rho_{2}\right) \\
& =m(k s-q r)-3 q \operatorname{frac}\left(\rho_{1}\right)-3(k-q) \operatorname{frac}\left(\rho_{2}\right) \\
& =\lambda m\left[\left(\begin{array}{c}
n-1 \\
3
\end{array}\right)-\left(\begin{array}{c}
m-1 \\
3
\end{array}\right)\right]-3 q \operatorname{frac}\left(\rho_{1}\right)-3(k-q) \operatorname{frac}\left(\rho_{2}\right),
\end{aligned}
$$

the right hand side inequality is equivalent to

$$
\lambda m\left(\begin{array}{c}
n-m \\
3
\end{array}\right)+2 \lambda\left(\begin{array}{c}
m \\
2
\end{array}\right)\left(\begin{array}{c}
n-m \\
2
\end{array}\right) \geqslant 3 q \operatorname{frac}\left(\rho_{1}\right)+3(k-q) \operatorname{frac}\left(\rho_{2}\right) .
$$

There are three cases to consider.

(a) $k>q, s>r$ : In this case $s \geqslant 2, n \geqslant 4 m / 3$. Since $\operatorname{frac}\left(\rho_{i}\right) \in\{0,1 / 3,2 / 3\}$ (for $\left.i=1,2\right)$, we have $3 q \operatorname{frac}\left(\rho_{1}\right)+3(k-q) \operatorname{frac}\left(\rho_{2}\right) \leqslant 2 k \leqslant \lambda\left(\begin{array}{c}n-1 \\ 3\end{array}\right)$. Therefore,

$$
\begin{aligned}
\alpha:= & \frac{6}{\lambda}\left[\lambda m\left(\begin{array}{c}
n-m \\
3
\end{array}\right)+2 \lambda\left(\begin{array}{c}
m \\
2
\end{array}\right)\left(\begin{array}{c}
n-m \\
2
\end{array}\right)-3 q \operatorname{frac}\left(\rho_{1}\right)-3(k-q) \operatorname{frac}\left(\rho_{2}\right)\right] \\
& \geqslant 6 m\left(\begin{array}{c}
n-m \\
3
\end{array}\right)+12\left(\begin{array}{c}
m \\
2
\end{array}\right)\left(\begin{array}{c}
n-m \\
2
\end{array}\right)-6\left(\begin{array}{c}
n-1 \\
3
\end{array}\right) \\
& =(m-1)\left(n^{3}-6 n^{2}+\left(-3 m^{2}+6 m+11\right) n+2 m^{3}-m^{2}-6 m-6\right) .
\end{aligned}
$$

Let $f(n)=n^{3}-6 n^{2}+\left(-3 m^{2}+6 m+11\right) n+2 m^{3}-m^{2}-6 m-6$, and $g(m)=$ $10 m^{3}-99 m^{2}+234 m-162$. Since $g(7)=55>0, g^{\prime}(7)=318>0, g^{\prime \prime}(7)=222>0$, we have that $g(m)>0$ for $m \geqslant 7$, and so, $f(4 m / 3)=g(m) / 27>0$ for $m \geqslant 7$. Moreover, $f^{\prime}(4 m / 3)=\left(7 m^{2}-30 m+33\right) / 3>0$ and $f^{\prime \prime}(4 m / 3)=8 m-12>0$ for $m \geqslant 7$. We conclude that $f(n) \geqslant 0$ for $n \geqslant 4 m / 3$ and $m \geqslant 7$. Thus, $\alpha \geqslant 0$ for $m \geqslant 7, n \geqslant 4 m / 3$. 
Recall that if $m=4$, then $r \geqslant 2$. For $m=5,6$, since $(m, r, \lambda)$ is admissible, we have $4 \mid \mathrm{rm}$. Consequently, for $m=4,5,6, s>r \geqslant 2$. We have

$$
\begin{array}{r}
\alpha \geqslant 6 m\left(\begin{array}{c}
n-m \\
3
\end{array}\right)+12\left(\begin{array}{c}
m \\
2
\end{array}\right)\left(\begin{array}{c}
n-m \\
2
\end{array}\right)-4\left(\begin{array}{c}
n-1 \\
3
\end{array}\right) \\
= \begin{cases}\frac{1}{3}\left(10 n^{3}-60 n^{2}-106 n+732\right) & \text { if } m=4, \\
\frac{1}{3}\left(13 n^{3}-78 n^{2}-397 n+2262\right) & \text { if } m=5, \\
\frac{8}{3}\left(2 n^{3}-12 n^{2}-113 n+663\right) & \text { if } m=6 .\end{cases}
\end{array}
$$

If $h(n)=10 n^{3}-60 n^{2}-106 n+732, k(n)=13 n^{3}-78 n^{2}-397 n+2262$, and $\ell(n)=$ $2 n^{3}-12 n^{2}-113 n+663$, then $h(6)=96>0, h^{\prime}(6)=254>0, h^{\prime \prime}(6)=240>0, k(7)=$ $120>0, k^{\prime}(7)=422>0, k^{\prime \prime}(7)=390>0, \ell(8)=15>0, \ell^{\prime}(8)=79>0, \ell^{\prime \prime}(8)=$ $72>0$. We conclude that $k(n) \geqslant 0$ for $n \geqslant 7$ and $\ell(n) \geqslant 0$ for $n \geqslant 8$. Thus, $\alpha \geqslant 0$ for $m=4, n \geqslant 6, m=5, n \geqslant 7$ and $m=6, n \geqslant 8$.

(b) $r=s$ : In this case $n \geqslant 2 m, \rho_{1}=0$. We have $3 q \operatorname{frac}\left(\rho_{1}\right)+3(k-q) \operatorname{frac}\left(\rho_{2}\right) \leqslant 2(k-q) \leqslant$ $2 \lambda\left[\left(\begin{array}{c}n-1 \\ 3\end{array}\right)-\left(\begin{array}{c}m-1 \\ 3\end{array}\right)\right]$. Therefore,

$$
\begin{aligned}
& \frac{6}{\lambda}\left(\lambda m\left(\begin{array}{c}
n-m \\
3
\end{array}\right)+2 \lambda\left(\begin{array}{c}
m \\
2
\end{array}\right)\left(\begin{array}{c}
n-m \\
2
\end{array}\right)-3 q \operatorname{frac}\left(\rho_{1}\right)-3(k-q) \operatorname{frac}\left(\rho_{2}\right)\right) \\
& \quad \geqslant 6 m\left(\begin{array}{c}
n-m \\
3
\end{array}\right)+12\left(\begin{array}{c}
m \\
2
\end{array}\right)\left(\begin{array}{c}
n-m \\
2
\end{array}\right)-12\left(\begin{array}{c}
n-1 \\
3
\end{array}\right)+12\left(\begin{array}{c}
m-1 \\
3
\end{array}\right) \\
& \quad=(m-2)(n-m)\left(n^{2}+m n-6 n-2 m^{2}-3 m+11\right) \\
& \quad>n(n-6)-3 m+11+m(n-2 m) \\
& \geqslant 4 n-3 m+11>0 .
\end{aligned}
$$

(c) $k=q$ : Since $3 q \operatorname{frac}\left(\rho_{1}\right)+3(k-q) \operatorname{frac}\left(\rho_{2}\right)=3 q \operatorname{frac}\left(\rho_{1}\right) \in\{0, q, 2 q\}$, we have

$$
\lambda m\left(\begin{array}{c}
n-m \\
3
\end{array}\right)+2 \lambda\left(\begin{array}{c}
m \\
2
\end{array}\right)\left(\begin{array}{c}
n-m \\
2
\end{array}\right) \geqslant \begin{cases}0 & \text { if } m(s-r) \equiv 0(\bmod 3), \\
q & \text { if } m(s-r) \equiv 1(\bmod 3), \\
2 q & \text { if } m(s-r) \equiv 2(\bmod 3),\end{cases}
$$

where the case $m(s-r) \equiv 0(\bmod 3)$ is trivial, and the remaining two cases are implied by (N8).

Lemma 6.2. If $s<3 r / 2$, then $k>q$.

Proof. Suppose on the contrary that $k=q$, which implies $s=r\left(\begin{array}{c}n-1 \\ 3\end{array}\right) /\left(\begin{array}{c}m-1 \\ 3\end{array}\right)$. Since $s<3 r / 2$, we have $2\left(\begin{array}{c}n-1 \\ 3\end{array}\right)<3\left(\begin{array}{c}m-1 \\ 3\end{array}\right)$, or equivalently, $2(n-1)(n-2)(n-3)<3(m-1)(m-2)(m-3)$. Because $n \geqslant 4 m / 3$, we have

$$
\begin{aligned}
2(n-1) & (n-2)(n-3)-3(m-1)(m-2)(m-3) \\
& \geqslant \frac{1}{9}\left(32 m^{2}-72 m+36\right)(4 m / 3-3)-\left(3 m^{2}-9 m+6\right)(m-3) \\
& \geqslant 0
\end{aligned}
$$

which is a contradiction. 


\section{Lemma 6.3.}

$$
e \leqslant \begin{cases}q\left\lfloor\rho_{1}^{\prime}\right\rfloor+(k-q)\left\lfloor\rho_{2}^{\prime}\right\rfloor & \text { if } \iota_{2} \geqslant 0, \rho_{1}^{\prime} \geqslant 0 \\ (k-q)\left\lfloor\rho_{2}^{\prime}\right\rfloor & \text { if } \iota_{2}>0, \rho_{1}^{\prime}<0 .\end{cases}
$$

Proof. First, let us assume that $\iota_{2} \geqslant 0, \rho_{1}^{\prime} \geqslant 0$. In this case, $n \leqslant 2 m$. If $n<2 m$, then $s>r$, and so $s \geqslant 2$. Since frac $\left(\rho_{i}^{\prime}\right) \in\{0,1 / 2\}$ (for $i=1,2$ ), we have $q \operatorname{frac}\left(\rho_{1}^{\prime}\right)+(k-q) \operatorname{frac}\left(\rho_{2}^{\prime}\right) \leqslant$ $k / 2 \leqslant \lambda\left(\begin{array}{c}n-1 \\ 3\end{array}\right) / 4$. We have

$$
\begin{aligned}
2 q\left\lfloor\rho_{1}^{\prime}\right\rfloor+2(k-q)\left\lfloor\rho_{2}^{\prime}\right\rfloor= & 2 q \rho_{1}^{\prime}+2(k-q) \rho_{2}^{\prime}-2 q \operatorname{frac}\left(\rho_{1}^{\prime}\right)-2(k-q) \operatorname{frac}\left(\rho_{2}^{\prime}\right) \\
= & k s m-\frac{3 q r m}{4}-\frac{k s n}{4}-2 q \operatorname{frac}\left(\rho_{1}^{\prime}\right)-2(k-q) \operatorname{frac}\left(\rho_{2}^{\prime}\right) \\
= & \lambda m\left(\begin{array}{c}
n-1 \\
3
\end{array}\right)-3 \lambda\left(\begin{array}{c}
m \\
4
\end{array}\right)-\lambda\left(\begin{array}{c}
n \\
4
\end{array}\right) \\
& -2 q \operatorname{frac}\left(\rho_{1}^{\prime}\right)-2(k-q) \operatorname{frac}\left(\rho_{2}^{\prime}\right) \\
\geqslant & \lambda m\left(\begin{array}{c}
n-1 \\
3
\end{array}\right)-3 \lambda\left(\begin{array}{c}
m \\
4
\end{array}\right)-\lambda\left(\begin{array}{c}
n \\
4
\end{array}\right)-\frac{\lambda}{2}\left(\begin{array}{c}
n-1 \\
3
\end{array}\right),
\end{aligned}
$$

and hence, to prove the first inequality, it suffices to show that

$$
\lambda m\left(\begin{array}{c}
n-1 \\
3
\end{array}\right)-3 \lambda\left(\begin{array}{c}
m \\
4
\end{array}\right)-\lambda\left(\begin{array}{c}
n \\
4
\end{array}\right)-\frac{\lambda}{2}\left(\begin{array}{c}
n-1 \\
3
\end{array}\right) \geqslant 2 \lambda(n-m)\left(\begin{array}{c}
m \\
3
\end{array}\right) .
$$

Therefore,

$$
\begin{aligned}
\alpha & :=\frac{12}{\lambda}\left[\lambda m\left(\begin{array}{c}
n-1 \\
3
\end{array}\right)-3 \lambda\left(\begin{array}{c}
m \\
4
\end{array}\right)-\lambda\left(\begin{array}{c}
n \\
4
\end{array}\right)-\frac{\lambda}{2}\left(\begin{array}{c}
n-1 \\
3
\end{array}\right)-2 \lambda(n-m)\left(\begin{array}{c}
m \\
3
\end{array}\right)\right] \\
= & 12 m\left(\begin{array}{c}
n-1 \\
3
\end{array}\right)-36\left(\begin{array}{c}
m \\
4
\end{array}\right)-12\left(\begin{array}{l}
n \\
4
\end{array}\right)-24(n-m)\left(\begin{array}{c}
m \\
3
\end{array}\right)-6\left(\begin{array}{c}
n-1 \\
3
\end{array}\right) \\
= & 12\left(\begin{array}{c}
m \\
2
\end{array}\right)\left(\begin{array}{c}
n-m \\
2
\end{array}\right)-6\left(\begin{array}{c}
m-1 \\
3
\end{array}\right)-6(n-m)\left(\begin{array}{c}
m-1 \\
2
\end{array}\right) \\
& -6(m-1)\left(\begin{array}{c}
n-m \\
2
\end{array}\right)-6\left(\begin{array}{c}
n-m \\
3
\end{array}\right)-12\left(\begin{array}{c}
n-m \\
4
\end{array}\right) \\
= & A+B+C+D,
\end{aligned}
$$

where $A=2\left(\begin{array}{c}m \\ 2\end{array}\right)\left(\begin{array}{c}n-m \\ 2\end{array}\right)-2\left(\begin{array}{c}n-m \\ 2\end{array}\right)\left(\begin{array}{c}n-m-2 \\ 2\end{array}\right), B=2\left(\begin{array}{c}m \\ 2\end{array}\right)\left(\begin{array}{c}n-m \\ 2\end{array}\right)-6(m-1)\left(\begin{array}{c}n-m \\ 2\end{array}\right), C=\left(\begin{array}{c}m \\ 2\end{array}\right)\left(\begin{array}{c}n-m \\ 2\end{array}\right)-$ $2(n-m-2)\left(\begin{array}{c}n-m \\ 2\end{array}\right)$, and $D=7\left(\begin{array}{c}m \\ 2\end{array}\right)\left(\begin{array}{c}n-m \\ 2\end{array}\right)-6(n-m)\left(\begin{array}{c}m-1 \\ 2\end{array}\right)-6\left(\begin{array}{c}m-1 \\ 3\end{array}\right)$.

Now, suppose that $m \geqslant 10$. Since $n \geqslant 4 m / 3$, we have $n-m \geqslant m / 3 \geqslant 4$. Since $n \leqslant 2 m$, we have $\left(\begin{array}{c}m \\ 2\end{array}\right) \geqslant\left(\begin{array}{c}n-m \\ 2\end{array}\right)$, and so $A \geqslant 0$. Since $m \geqslant 6$, we have $4\left(\begin{array}{c}m \\ 2\end{array}\right) \geqslant 12(m-1)$, and hence, $B \geqslant 0$. Moreover, $n \leqslant 2 m$ implies that $\left(\begin{array}{c}m \\ 2\end{array}\right) \geqslant 2(n-m-2)$, which means that $C \geqslant 0$. Finally,

$$
D=\frac{1}{2}(m-1)\left(7 m n^{2}+\left(-14 m^{2}-19 m+24\right) n+7 m^{3}+15 m^{2}-4 m-24\right) .
$$

Let $f(n)=7 m n^{2}+\left(-14 m^{2}-19 m+24\right) n+7 m^{3}+15 m^{2}-4 m-24$ and $g(m)=7 m^{3}-$ $93 m^{2}+252 m-216$. Since $g(10)=4>0, g^{\prime}(10)=492>0$, and $g^{\prime \prime}(10)=234>0$, we have $f(4 m / 3)=g(m) / 9 \geqslant 0$, for $m \geqslant 10$. Moreover, $f^{\prime}(4 m / 3)=\frac{1}{3}\left(14 m^{2}-57 m+72\right) \geqslant 0$, for $m \geqslant 10$. Therefore, $D \geqslant 0$, for $m \geqslant 10$, which implies that $\alpha \geqslant 0$ for $m \geqslant 10$. 
For $m=7,8,9$, we have $\alpha \geqslant 24 m\left(\begin{array}{c}n-1 \\ 3\end{array}\right)-72\left(\begin{array}{c}m \\ 4\end{array}\right)-24\left(\begin{array}{c}n \\ 4\end{array}\right)-48(n-m)\left(\begin{array}{c}m \\ 3\end{array}\right)-12\left(\begin{array}{c}n-1 \\ 3\end{array}\right)$, which is nonnegative for $4 m / 3 \leqslant n \leqslant 2 m$.

For $m=4$, we have $s \geqslant 3$. For $m=5,6$, since $(m, r, \lambda)$ is admissible, we have $4 \mid \mathrm{rm}$. Consequently, $r \geqslant 2$ and so $s \geqslant 3$. Hence, we have $\alpha \geqslant 24 m\left(\begin{array}{c}n-1 \\ 3\end{array}\right)-72\left(\begin{array}{c}m \\ 4\end{array}\right)-24\left(\begin{array}{c}n \\ 4\end{array}\right)-48(n-$ $m)\left(\begin{array}{c}m \\ 3\end{array}\right)-8\left(\begin{array}{c}n-1 \\ 3\end{array}\right)$, which is nonnegative for $m=4,5,6,4 m / 3 \leqslant n \leqslant 2 m$.

If $n=2 m$, then $q \operatorname{frac}\left(\rho_{1}^{\prime}\right)+(k-q) \operatorname{frac}\left(\rho_{2}^{\prime}\right) \leqslant k / 2 \leqslant \lambda\left(\begin{array}{c}n-1 \\ 3\end{array}\right) / 2$. Therefore, we need to show that

$$
\lambda m\left(\begin{array}{c}
2 m-1 \\
3
\end{array}\right)-3 \lambda\left(\begin{array}{c}
m \\
4
\end{array}\right)-\lambda\left(\begin{array}{c}
2 m \\
4
\end{array}\right)-\lambda\left(\begin{array}{c}
2 m-1 \\
3
\end{array}\right) \geqslant 2 \lambda(2 m-m)\left(\begin{array}{c}
m \\
3
\end{array}\right)
$$

which can be simplified as the inequality $(5 m-3)(m-1)(m-2)(m-4) / 24 \geqslant 0$, which holds for $m \geqslant 4$.

Now, let us assume that $\iota_{2} \geqslant 0, \rho_{1}^{\prime} \leqslant 0$. In this case, $r<s<3 r / 2$, and $(4-3 r / s) m<$ $n<2 m$. Therefore, $s \geqslant 4$, and $s / r<3 m /(4 m-n)$. It is also clear that $r \leqslant \lambda\left(\begin{array}{c}m-1 \\ 3\end{array}\right)$. Since $\operatorname{frac}\left(\rho_{2}^{\prime}\right) \in\{0,1 / 2\}$, we have

$$
8(k-q) \operatorname{frac}\left(\rho_{2}^{\prime}\right) \leqslant \frac{4 \lambda}{s}\left(\begin{array}{c}
n-1 \\
3
\end{array}\right)-\frac{4 \lambda}{r}\left(\begin{array}{c}
m-1 \\
3
\end{array}\right) \leqslant \lambda\left(\begin{array}{c}
n-1 \\
3
\end{array}\right)-4 .
$$

Therefore,

$$
\begin{aligned}
8(k-q)\left\lfloor\rho_{2}^{\prime}\right\rfloor & =8(k-q) \rho_{2}^{\prime}-8(k-q) \operatorname{frac}\left(\rho_{2}^{\prime}\right) \\
& =4 k s m-k s n-4 q s m+q s n-8(k-q) \operatorname{frac}\left(\rho_{2}^{\prime}\right) \\
& =(4 m-n)(k s-q s)-8(k-q) \operatorname{frac}\left(\rho_{2}^{\prime}\right) \\
& =\lambda(4 m-n)\left[\left(\begin{array}{c}
n-1 \\
3
\end{array}\right)-\frac{s}{r}\left(\begin{array}{c}
m-1 \\
3
\end{array}\right)\right]-8(k-q) \operatorname{frac}\left(\rho_{2}^{\prime}\right) \\
& \geqslant \lambda(4 m-n)\left[\left(\begin{array}{c}
n-1 \\
3
\end{array}\right)-\frac{s}{r}\left(\begin{array}{c}
m-1 \\
3
\end{array}\right)\right]-\lambda\left(\begin{array}{c}
n-1 \\
3
\end{array}\right)+4 .
\end{aligned}
$$

Therefore, it suffices to show that

$$
\lambda(4 m-n)\left[\left(\begin{array}{c}
n-1 \\
3
\end{array}\right)-\frac{s}{r}\left(\begin{array}{c}
m-1 \\
3
\end{array}\right)\right]-\lambda\left(\begin{array}{c}
n-1 \\
3
\end{array}\right)+4 \geqslant 8 \lambda(n-m)\left(\begin{array}{c}
m \\
3
\end{array}\right) .
$$

Hence,

$$
\begin{aligned}
\alpha & :=(4 m-n)\left[\left(\begin{array}{c}
n-1 \\
3
\end{array}\right)-\frac{s}{r}\left(\begin{array}{c}
m-1 \\
3
\end{array}\right)\right]-8(n-m)\left(\begin{array}{c}
m \\
3
\end{array}\right)-\left(\begin{array}{c}
n-1 \\
3
\end{array}\right)+4 / \lambda \\
\geqslant & (4 m-n)\left(\begin{array}{c}
n-1 \\
3
\end{array}\right)-3 m\left(\begin{array}{c}
m-1 \\
3
\end{array}\right)-8(n-m)\left(\begin{array}{c}
m \\
3
\end{array}\right)-\left(\begin{array}{c}
n-1 \\
3
\end{array}\right) \\
\geqslant & 4\left(\begin{array}{c}
m \\
2
\end{array}\right)\left(\begin{array}{c}
n-m \\
2
\end{array}\right)-(n-m)\left(\begin{array}{c}
m-1 \\
2
\end{array}\right)-4\left(\begin{array}{c}
n-m \\
4
\end{array}\right) \\
& -\left(\begin{array}{c}
m-1 \\
3
\end{array}\right)-(m-1)\left(\begin{array}{c}
n-m \\
2
\end{array}\right)-\left(\begin{array}{c}
n-m \\
3
\end{array}\right) \\
= & A+B,
\end{aligned}
$$

where $A=3\left(\begin{array}{c}m \\ 2\end{array}\right)\left(\begin{array}{c}n-m \\ 2\end{array}\right)-(n-m)\left(\begin{array}{c}m-1 \\ 2\end{array}\right)$ and $B=\left(\begin{array}{c}m \\ 2\end{array}\right)\left(\begin{array}{c}n-m \\ 2\end{array}\right)-4\left(\begin{array}{c}n-m \\ 4\end{array}\right)-\left(\begin{array}{c}m-1 \\ 3\end{array}\right)-(m-$ 1) $\left(\begin{array}{c}n-m \\ 2\end{array}\right)-\left(\begin{array}{c}n-m \\ 3\end{array}\right)$. Since $3\left(\begin{array}{c}n-m \\ 2\end{array}\right) \geqslant n-m$ and $\left(\begin{array}{c}m \\ 2\end{array}\right) \geqslant\left(\begin{array}{c}m-1 \\ 2\end{array}\right), A$ is non-negative. We have $B=\left(\begin{array}{c}m-1 \\ 2\end{array}\right)\left[3\left(\begin{array}{c}n-m \\ 2\end{array}\right)-m+3\right] / 3-\left(\begin{array}{c}n-m \\ 2\end{array}\right)\left(\begin{array}{c}n-m-1 \\ 2\end{array}\right) / 3$. Since $n \leqslant 2 m-1$, we have 
$\left(\begin{array}{c}m-1 \\ 2\end{array}\right) \geqslant\left(\begin{array}{c}n-m \\ 2\end{array}\right)$. So, it is enough to show that $3\left(\begin{array}{c}n-m \\ 2\end{array}\right)-m+3 \geqslant\left(\begin{array}{c}n-m-1 \\ 2\end{array}\right)$, or equivalently, $n^{2}-2 m n+m^{2}-m+2 \geqslant 0$. Let $f(n)=n^{2}-2 m n+m^{2}-m+2$. Since $f(4 m / 3)=(m-3)(m-6) / 9 \geqslant 0$ and $f^{\prime}(4 m / 3)=2 m / 3 \geqslant$ for $m \geqslant 6$, we have $B \geqslant 0$, for $m \geqslant 6$. For $m=4,5$, we have

$$
\alpha \geqslant(4 m-n)\left(\begin{array}{c}
n-1 \\
3
\end{array}\right)-3 m\left(\begin{array}{c}
m-1 \\
3
\end{array}\right)-8(n-m)\left(\begin{array}{c}
m \\
3
\end{array}\right)-\left(\begin{array}{c}
n-1 \\
3
\end{array}\right),
$$

which is nonnegative for $m=4,5$ and $4 m / 3 \leqslant n \leqslant 2 m-1$.

Lemma 6.4. If all the $u^{3} v$-edges are colored, then

$$
f \geqslant\left\{\begin{array}{l}
\sum_{j \in \kappa_{1}} \iota_{1 j}+\sum_{j \in \kappa_{2}} \iota_{2 j} \\
\sum_{j \in \kappa_{2}} \iota_{2 j}
\end{array} \quad \text { if } e_{j}=0 \text { for } j \in \kappa_{1} .\right.
$$

Proof. We have

$$
\begin{aligned}
\lambda\left(\begin{array}{c}
m \\
2
\end{array}\right) & \left(\begin{array}{c}
n-m \\
2
\end{array}\right)-\sum_{j \in \kappa_{1}} \iota_{1 j}-\sum_{j \in \kappa_{2}} \iota_{2 j} \\
= & \lambda\left(\begin{array}{c}
m \\
2
\end{array}\right)\left(\begin{array}{c}
n-m \\
2
\end{array}\right)-k\left(s m-\frac{s n}{4}\right)+q \frac{3 r m}{4}+2 \lambda(n-m)\left(\begin{array}{c}
m \\
3
\end{array}\right) \\
= & \lambda\left(\begin{array}{c}
m \\
2
\end{array}\right)\left(\begin{array}{c}
n-m \\
2
\end{array}\right)-\lambda m\left(\begin{array}{c}
n-1 \\
3
\end{array}\right)+\lambda\left(\begin{array}{c}
n \\
4
\end{array}\right)+3 \lambda\left(\begin{array}{c}
m \\
4
\end{array}\right)+2 \lambda(n-m)\left(\begin{array}{c}
m \\
3
\end{array}\right) \\
= & \lambda\left(\begin{array}{c}
n-m \\
4
\end{array}\right) \geqslant 0 .
\end{aligned}
$$

Now suppose that $e_{j}=0$ for $j \in \kappa_{1}$. We have

$$
\begin{aligned}
\lambda\left(\begin{array}{c}
m \\
2
\end{array}\right) & \left(\begin{array}{c}
n-m \\
2
\end{array}\right)-\sum_{j \in \kappa_{2}} \iota_{2 j} \\
= & \lambda\left(\begin{array}{c}
m \\
2
\end{array}\right)\left(\begin{array}{c}
n-m \\
2
\end{array}\right)-(k-q)\left(s m-\frac{s n}{4}\right)+2 \sum_{j \in \kappa_{2}} e_{j} \\
= & \lambda\left(\begin{array}{c}
m \\
2
\end{array}\right)\left(\begin{array}{c}
n-m \\
2
\end{array}\right)+2 \lambda(n-m)\left(\begin{array}{c}
m \\
3
\end{array}\right)-(k-q)\left(s m-\frac{s n}{4}\right) \geqslant 0,
\end{aligned}
$$

where the last inequality holds by (N7).

Lemma 6.5. We can color the $u^{3} v$-edges so that

$$
f \leqslant \sum_{j \in \kappa_{1}}\left\lfloor\rho_{1 j}\right\rfloor+\sum_{j \in \kappa_{2}}\left\lfloor\rho_{2 j}\right\rfloor .
$$

Proof. We prove something stronger. We show that in most cases as long as all the $u^{3} v$-edges are colored, the above inequality holds. In the remaining few cases, we color the $u^{3} v$-edges carefully ensuring that certain conditions are met (see Table 3$)$. Since $(m, r, \lambda)$ is admissible, $4 \mid \mathrm{rm}$. Therefore,

$$
\operatorname{frac}\left(\rho_{i j}\right)=\left\{\begin{array}{c}
0 \text { if } s m+\operatorname{mult}_{j}\left(u^{3} v\right) \equiv 0(\bmod 2) \\
\frac{1}{2} \text { if } s m+\operatorname{mult}_{j}\left(u^{3} v\right) \equiv 1(\bmod 2)
\end{array}\right\} \quad j \in \kappa_{i}, i=1,2
$$


Let $\beta=\sum_{j \in \kappa_{1}} \operatorname{frac}\left(\rho_{1 j}\right)+\sum_{j \in \kappa_{2}} \operatorname{frac}\left(\rho_{2 j}\right)$. Then

$$
2 \beta=\left|\left\{j \in \kappa: s m+\operatorname{mult}_{j}\left(u^{3} v\right) \equiv 1(\bmod 2)\right\}\right| \leqslant k
$$

We have

$$
\begin{aligned}
& 2 \sum_{j \in \kappa_{1}}\left\lfloor\rho_{1 j}\right\rfloor+2 \sum_{j \in \kappa_{2}}\left\lfloor\rho_{2 j}\right\rfloor-2 \lambda\left(\begin{array}{c}
m \\
2
\end{array}\right)\left(\begin{array}{c}
n-m \\
2
\end{array}\right) \\
& \quad=2 \sum_{j \in \kappa_{1}} \rho_{1 j}+2 \sum_{j \in \kappa_{2}} \rho_{2 j}-2 \lambda\left(\begin{array}{c}
m \\
2
\end{array}\right)\left(\begin{array}{c}
n-m \\
2
\end{array}\right)-2 \beta \\
& \quad=q m(s-r)+(k-q) s m-3 \lambda(n-m)\left(\begin{array}{c}
m \\
3
\end{array}\right)-2 \lambda\left(\begin{array}{c}
m \\
2
\end{array}\right)\left(\begin{array}{c}
n-m \\
2
\end{array}\right)-2 \beta \\
& \quad=k s m-q r m-3 \lambda(n-m)\left(\begin{array}{c}
m \\
3
\end{array}\right)-2 \lambda\left(\begin{array}{c}
m \\
2
\end{array}\right)\left(\begin{array}{c}
n-m \\
2
\end{array}\right)-2 \beta \\
& =\lambda m\left[\left(\begin{array}{c}
n-1 \\
3
\end{array}\right)-\left(\begin{array}{c}
m-1 \\
3
\end{array}\right)\right]-3 \lambda(n-m)\left(\begin{array}{c}
m \\
3
\end{array}\right)-2 \lambda\left(\begin{array}{c}
m \\
2
\end{array}\right)\left(\begin{array}{c}
n-m \\
2
\end{array}\right)-2 \beta \\
& =\lambda m\left(\begin{array}{c}
n-m \\
3
\end{array}\right)-2 \beta .
\end{aligned}
$$

First, let us assume that $n \geqslant 3 m$. We have

$$
\begin{aligned}
\alpha & :=\frac{3}{\lambda}\left[\lambda m\left(\begin{array}{c}
n-m \\
3
\end{array}\right)-2 \beta\right] \\
& \geqslant \frac{3}{\lambda}\left[\lambda m\left(\begin{array}{c}
n-m \\
3
\end{array}\right)-k\right] \\
& \geqslant 3 m\left(\begin{array}{c}
n-m \\
3
\end{array}\right)-3\left(\begin{array}{c}
n-1 \\
3
\end{array}\right) \\
& =3 m\left(\begin{array}{c}
n-m \\
3
\end{array}\right)-3\left(\begin{array}{c}
m-1 \\
3
\end{array}\right)-3(n-m)\left(\begin{array}{c}
m-1 \\
2
\end{array}\right)-3(m-1)\left(\begin{array}{c}
n-m \\
2
\end{array}\right)-3\left(\begin{array}{c}
n-m \\
3
\end{array}\right) \\
& =(m-1)(n-m-5)\left(\begin{array}{c}
n-m \\
2
\end{array}\right)-(4 m-3 n-3)\left(\begin{array}{c}
m-1 \\
2
\end{array}\right) .
\end{aligned}
$$

Since $n \geqslant 3 m$, we have $\left(\begin{array}{c}n-m \\ 2\end{array}\right) \geqslant\left(\begin{array}{c}m-1 \\ 2\end{array}\right)$ and $(m-1)(n-m-5) \geqslant 4 m-3 n-3$. This implies $\alpha \geqslant 0$.

Now, let us assume that $4 m / 3 \leqslant n \leqslant 3 m, s \geqslant 2$, and $m \geqslant 38$. Since $s \geqslant 2$, we have $2 k \leqslant \lambda\left(\begin{array}{c}n-1 \\ 3\end{array}\right)$. Therefore,

$$
\begin{aligned}
4 \alpha & \geqslant 12 m\left(\begin{array}{c}
n-m \\
3
\end{array}\right)-6\left(\begin{array}{c}
n-1 \\
3
\end{array}\right) \\
& =12 m\left(\begin{array}{c}
n-m \\
3
\end{array}\right)-6\left[\left(\begin{array}{c}
m-1 \\
3
\end{array}\right)+(n-m)\left(\begin{array}{c}
m-1 \\
2
\end{array}\right)+(m-1)\left(\begin{array}{c}
n-m \\
2
\end{array}\right)+\left(\begin{array}{c}
n-m \\
3
\end{array}\right)\right] \\
& =6(2 m-1)\left(\begin{array}{c}
n-m \\
3
\end{array}\right)-6\left(\begin{array}{c}
m-1 \\
3
\end{array}\right)-6(n-m)\left(\begin{array}{c}
m-1 \\
2
\end{array}\right)-6(m-1)\left(\begin{array}{c}
n-m \\
2
\end{array}\right) \\
& \geqslant(m-1)\left(2 n^{3}-3(2 m+3) n^{2}+\left(6 m^{2}+15 m+13\right) n-2 m^{3}-7 m^{2}-8 m-6\right) .
\end{aligned}
$$


Let $f(n)=2 n^{3}-3(2 m+3) n^{2}+\left(6 m^{2}+15 m+13\right) n-2 m^{3}-7 m^{2}-8 m-6, g(m)=$ $2 m^{3}-81 m^{2}+252 m-162$, and $h(m)=2 m^{3}-13 m^{2}+18 m-6$. Since $g(38)=2194>$ $0, g^{\prime}(38)=2760>0, g^{\prime \prime}(38)=294>0$, we have that $g(m)>0$ for $m \geqslant 38$, and so, $f(4 m / 3)=g(m) / 27>0$ for $m \geqslant 38$. Moreover, $f^{\prime}(4 m / 3)=\left(2 m^{2}-27 m+39\right) / 3>0$ and $f^{\prime \prime}(4 m / 3)=4 m-18>0$ for $m \geqslant 38$. We conclude that $f(n) \geqslant 0$ for $n \geqslant 4 m / 3$ and $m \geqslant 38$. Thus, $\alpha \geqslant 0$ for $m \geqslant 38, n \geqslant 4 m / 3$.

Now, let us assume that $4 m / 3 \leqslant n \leqslant 3 m, r=s=1$, and $m \geqslant 29$. Since $r=s$, we have $n \geqslant 2 m$. Therefore,

$$
\begin{aligned}
\alpha & \geqslant \frac{6}{\lambda}\left[\lambda m\left(\begin{array}{c}
n-m \\
3
\end{array}\right)-k\right] \\
& \geqslant 6 m\left(\begin{array}{c}
n-m \\
3
\end{array}\right)-6\left(\begin{array}{c}
n-1 \\
3
\end{array}\right) \\
& \geqslant 6 m\left(\begin{array}{c}
m \\
3
\end{array}\right)-6\left(\begin{array}{c}
3 m-1 \\
3
\end{array}\right) \\
& =(m-1)\left[m^{2}(m-2)-3(3 m-1)(3 m-2)\right] \\
& \geqslant(m-1)\left[m^{2}(m-2)-9 m(3 m-2)\right] \\
& =m(m-1)\left(m^{2}-29 m+18\right) \\
& \geqslant 0 .
\end{aligned}
$$

For the remaining cases, we refer the reader to Table 3.

Lemma 6.6. If $\iota_{2} \leqslant 0$ and $\rho_{2}^{\prime} \geqslant 0$, then $f \geqslant k$.

Proof. Since $\iota_{2} \leqslant 0$ and $\rho_{2}^{\prime} \geqslant 0$, we have $2 m \leqslant n \leqslant 4 m$. Now, we have

$$
\begin{aligned}
\frac{12}{\lambda}\left[\lambda\left(\begin{array}{c}
m \\
2
\end{array}\right)\left(\begin{array}{c}
n-m \\
2
\end{array}\right)-k\right]= & 12\left(\begin{array}{c}
m \\
2
\end{array}\right)\left(\begin{array}{c}
n-m \\
2
\end{array}\right)-\frac{12}{s}\left(\begin{array}{c}
n-1 \\
3
\end{array}\right) \\
\geqslant & 12\left(\begin{array}{c}
m \\
2
\end{array}\right)\left(\begin{array}{c}
n-m \\
2
\end{array}\right)-12\left(\begin{array}{c}
n-1 \\
3
\end{array}\right) \\
= & -2 n^{3}+\left(3 m^{2}-3 m+12\right) n^{2}+\left(-6 m^{3}+3 m^{2}+3 m-22\right) n \\
& +3 m^{4}-3 m^{2}+12 .
\end{aligned}
$$

Let $f(n)=-2 n^{3}+\left(3 m^{2}-3 m+12\right) n^{2}+\left(-6 m^{3}+3 m^{2}+3 m-22\right) n+3 m^{4}-3 m^{2}+12, g(m)=$ $3 m^{2}-13 m+6, h(m)=27 m^{3}-164 m^{2}+201 m-88, k(m)=6 m^{3}-33 m^{2}+51 m-22, l(m)=$ $18 m^{3}-117 m^{2}+99 m-22$. Since $g(6)=36>0, g^{\prime}(6)=23>0, h(6)=1046>0, h^{\prime}(6)=$ $1149>0, h^{\prime \prime}(6)=644>0, k(6)=392>0, k^{\prime}(6)=303>0, k^{\prime \prime}(6)=150>0, l(6)=248>$ $0, l^{\prime}(6)=639>0, l^{\prime \prime}(6)=414>0$, we have $f(2 m)=(m-1)(m-2) g(m) \geqslant 0, f^{\prime}(2 m)=$ $k(m) \geqslant 0, f(4 m)=27 m^{4}-164 m^{3}+201 m^{2}-88 m+12>m h(m) \geqslant 0$, and $f^{\prime}(4 m)=l(m) \geqslant 0$ for $m \geqslant 6$.

If $m=5$, then by (1), $4 \mid 5 r$. Hence, $r \geqslant 4$, which implies $s \geqslant 4$. Moreover, if $m=4$, we have $r \geqslant 2$, which implies $s \geqslant 2$. Hence, we have 


$$
\begin{aligned}
& \frac{12}{\lambda}\left[\lambda\left(\begin{array}{c}
m \\
2
\end{array}\right)\left(\begin{array}{c}
n-m \\
2
\end{array}\right)-k\right] \geqslant 12\left(\begin{array}{c}
m \\
2
\end{array}\right)\left(\begin{array}{c}
n-m \\
2
\end{array}\right)-6\left(\begin{array}{c}
n-1 \\
3
\end{array}\right) \\
& = \begin{cases}-n^{3}+42 n^{2}-335 n+726 & \text { if } m=4 \\
-n^{3}+66 n^{2}-671 n+1806 & \text { if } m=5 .\end{cases}
\end{aligned}
$$

Let $f(n)=-n^{3}+42 n^{2}-335 n+726, g(n)=-n^{3}+66 n^{2}-671 n+1806$. Since $f(8)=222>$ $0, f^{\prime}(8)=145>0, f(16)=2022>0, f^{\prime}(16)=241>0, g(10)=696>0, g^{\prime}(10)=349>$ $0, g(20)=6786>0, g^{\prime}(20)=1849>0$, we have $\lambda\left(\begin{array}{c}m \\ 2\end{array}\right)\left(\begin{array}{c}n-m \\ 2\end{array}\right)-k \geqslant 0$, for $m=4,5,2 m \leqslant n \leqslant$ $4 \mathrm{~m}$. This completes the proof.

\section{ACKNOWLEDGEMENT}

The authors wish to thank Anna Johnsen, Lana Kühle, Stefan Napirata, Songling Shan, and Lisa Zyga for their feedback on various aspects of this paper.

\section{REFERENCES}

[1] Amin Bahmanian. Detachments of hypergraphs I: The Berge-Johnson problem. Combin. Probab. Comput., 21(4):483-495, 2012.

[2] Amin Bahmanian and Mike Newman. Embedding factorizations for 3-uniform hypergraphs II: $r$ factorizations into $s$-factorizations. Electron. J. Combin., 23(2):Paper 2.42, 14, 2016.

[3] Amin Bahmanian and Mike Newman. Extending factorizations of complete uniform hypergraphs. Combinatorica, 38(6):1309-1335, 2018.

[4] Zs. Baranyai. On the factorization of the complete uniform hypergraph. In Infinite and finite sets (Colloq., Keszthely, 1973; dedicated to P. Erdős on his 60th birthday), Vol. I, pages 91-108. Colloq. Math. Soc. Jánōs Bolyai, Vol. 10. North-Holland, Amsterdam, 1975.

[5] Darryn Bryant and Daniel Horsley. A proof of Lindner's conjecture on embeddings of partial Steiner triple systems. J. Combin. Des., 17(1):63-89, 2009.

[6] Peter J. Cameron. Parallelisms of complete designs. Cambridge University Press, Cambridge-New YorkMelbourne, 1976. London Mathematical Society Lecture Note Series, No. 23.

[7] Allan B. Cruse. On embedding incomplete symmetric Latin squares. J. Combinatorial Theory Ser. A, 16:18-22, 1974.

[8] Stefan Glock, Daniela Kühn, Allan Lo, and Deryk Osthus. The existence of designs via iterative absorption: hypergraph $f$-designs for arbitrary $f, 2016$.

[9] R. Häggkvist and T. Hellgren. Extensions of edge-colourings in hypergraphs. I. In Combinatorics, Paul Erdős is eighty, Vol. 1, Bolyai Soc. Math. Stud., pages 215-238. János Bolyai Math. Soc., Budapest, 1993.

[10] Peter Keevash. The existence of designs. arXiv e-prints, page arXiv:1401.3665, Jan 2014.

[11] Charles C. Lindner and Trevor Evans. Finite embedding theorems for partial designs and algebras. Les Presses de l'Université de Montréal, Montreal, Que., 1977. Séminaire de Mathématiques Supérieures, No. 56 (Été 1971).

[12] C. A. Rodger and E. B. Wantland. Embedding edge-colorings into 2-edge-connected $k$-factorizations of $K_{k n+1}$. J. Graph Theory, 19(2):169-185, 1995.

Department of Mathematics, Illinois State University, Normal, IL USA 61790-4520

Department of Pathology and Laboratory Medicine, Western University, London, On, CANAdA, N6A 5C1

E-mail address: shaghsh@uwo.ca 


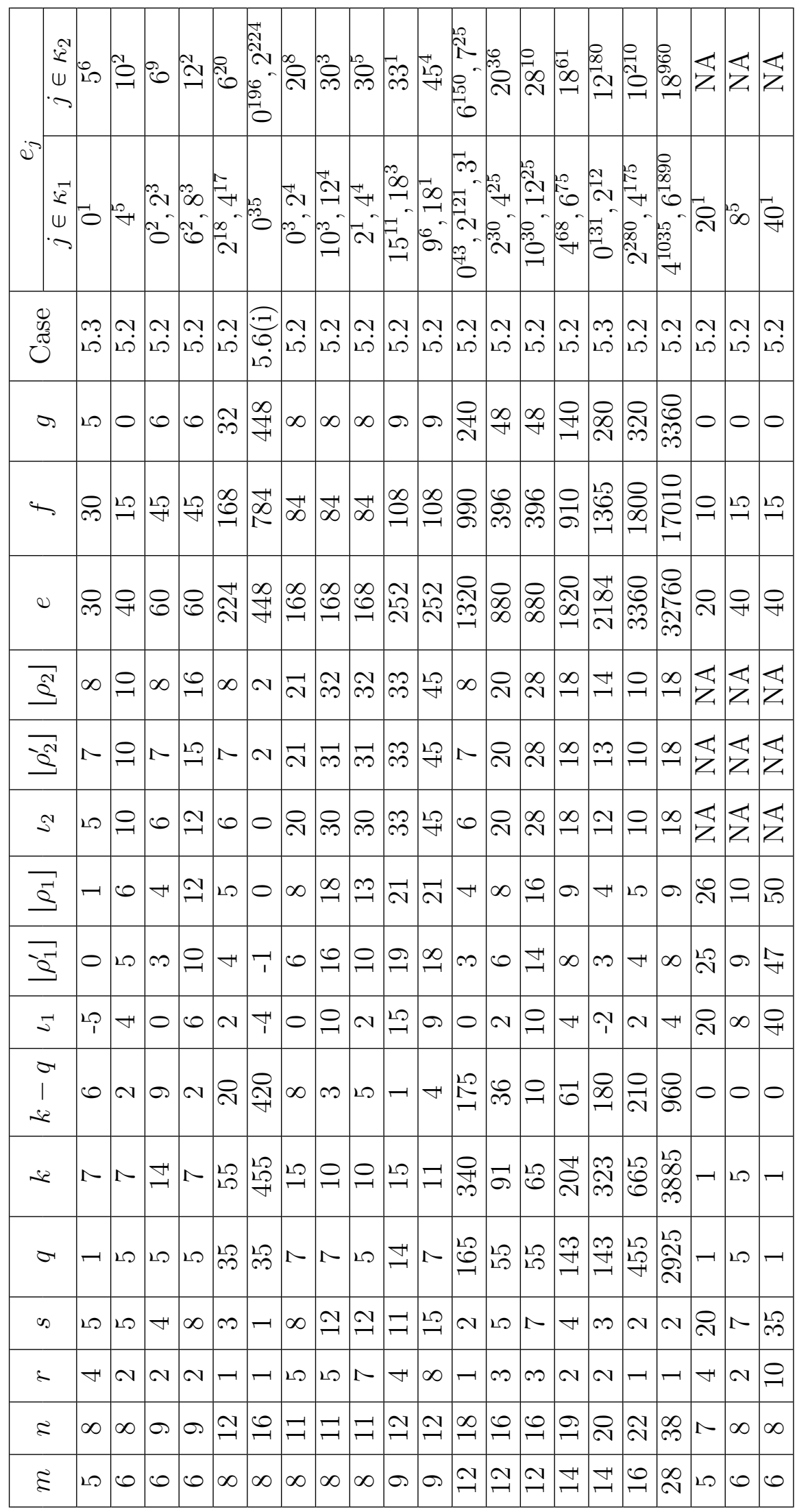

TABle 3. 